\title{
The averaging of gravity currents in porous media
}

\author{
Daniel M. Anderson ${ }^{\text {a) }}$ \\ Department of Mathematical Sciences, George Mason University, Fairfax, Virginia 22030
}

Richard M. McLaughlin ${ }^{\text {b) }}$

Department of Mathematics, University of North Carolina, Chapel Hill, North Carolina 27599

Cass T. Miller ${ }^{\mathrm{c}}$

Department of Environmental Sciences and Engineering, University of North Carolina, Chapel Hill, North Carolina 27599

(Received 19 December 2002; accepted 24 June 2003; published 15 August 2003)

\begin{abstract}
We explore the problem of a moving free surface in a water-saturated porous medium that has either a homogeneous or a periodically heterogeneous permeability field. We identify scaling relations and derive similarity solutions for the homogeneous, constant coefficient case in both a Cartesian and an axisymmetric, radial coordinate system. We utilize these similarity scalings to identify half-height slumping time scales as a rough guide for field groundwater cleanup strategies involving injected brines. We derive averaged solutions using homogenization for a vertically periodic, a horizontally periodic, and a two-dimensional periodic case-the solution of which requires solving a cell problem. Using effective coefficients, we connect the first two of these homogenized solutions to the similarity scaling solution derived for the homogeneous case. By simplifying to a thin limit, retaining variations of the porous media in the horizontal direction, we derive a homogenization solution in agreement with the general horizontally layered solution and an expression for the leading-order correction. Finally, we implement two numerical solution approaches and show that self-similar scaling and agreement with leading-order averaging emerge in finite time, and demonstrate the accuracy and convergence rate of the leading order correction for both the interior and the boundary of the domain. (C) 2003 American Institute of Physics.
\end{abstract}

[DOI: $10.1063 / 1.1600733$ ]

\section{INTRODUCTION}

Groundwater is an important resource, which is relied upon by about half of the population of the U.S. as a source of drinking water. Over the past few decades, releases of contaminants that are immiscible with water into the shallow subsurface has occurred in many instances, leading to contaminated conditions that pose a threat to human and ecological health. Due to capillary forces, such contaminants are trapped in the pore space of the porous media existing in the subsurface, leading to long-term sources of contamination. While much is known about such multiphase systems, many open questions still remain. ${ }^{1}$

Remediation of porous medium systems contaminated by nonaqueous phase liquids that are more dense than water (DNAPLs) are a particularly difficult class of problem, which motivate this investigation. Recently, Miller and coworkers have advanced a set of remediation strategies that rely upon the use of dense brine solutions to mobilize or contain DNAPL contaminants in porous medium systems. ${ }^{2,3}$ Since the brines used in these remediation technologies are significantly more dense than water and relatively expensive, the dynamics of brine behavior in porous medium systems

\footnotetext{
a)Telephone: (703) 993-1482; fax: (703) 993-1491. Electronic mail: danders1@gmu.edu

${ }^{b)}$ Electronic mail: rmm@amath.unc.edu

${ }^{c)}$ Electronic mail: casey_miller@unc.edu
}

are of interest for the economical assessment and design of such technologies. The complexities of the physics of density-dependent multiphase flow in heterogeneous porous medium systems makes this class of problem challenging. As a first step in our efforts to analyze this problem, we focus on the dynamics of a gravity-driven redistribution of a single dense fluid in heterogeneous porous medium.

Much is known about gravity-driven motion of a fluid mass in a uniform porous medium. Theoretical descriptions of the slumping of a groundwater mound and the historical development can be found in Bear ${ }^{4}$ and Barenblatt. ${ }^{5}$ Classical similarity solutions satisfying the Boussinesq equation (porous medium equation) have been a central part of this development. Extensions of these solutions have, for example, accounted for capillary effects in which some fluid is left behind in the draining region and not all pore space is occupied in the filling region (see Kochina et al. ${ }^{6}$ and Barenblatt ${ }^{5}$ ). Properties of similarity solutions of the classical porous medium equation and its close relatives have been carefully documented (e.g., Witelski and Bernoff ${ }^{7}$ ). The utility of these solutions in characterizing the long-time asymptotic behavior of fluid motion in spatially uniform porous media is well known. In the present work, we are interested in extending the utility of these free-boundary problem solutions using homogenization techniques to situations in 
which a spatially periodic background permeability variation is present.

There has been some recent work in which the homogenization of problems involving interfaces has been addressed. Friedman, $\mathrm{Hu}$, and $\mathrm{Liu}^{8}$ used homogenization theory for the Poisson equation on a domain with a rapidly oscillating structure along one of its boundaries and developed error formulas for these approximations. Friedman and $\mathrm{Hu}^{9}$ and Friedman ${ }^{10}$ applied similar analyses to a free-boundary problem for a chemical vapor deposition model that incorporated a rapidly varying periodic structure on the growing substrate. They described the existence and uniqueness properties for the homogenization approach and gave error estimates associated with the homogenized boundary.

Here we address the mathematical assessment of averaged time scales for the slumping of a gravity current in porous media, and the study of the ensuing interface shapes reflecting the underlying permeability properties. We will focus upon situations in which a fixed volume, elevated, localized brine layer is allowed to spread under the action of gravity in an isotropic, nonuniform permeability porous medium on top of an impermeable bottom layer.

The paper is organized as follows. In Sec. II, we overview the equations of motion for such a moving interface problem. In Sec. III, we review known similarity solutions for this system for the simplest case of a constant permeability tensor, ${ }^{4,5}$ and use these solutions to predict associated half-height slumping time scales for the gravity current. In Sec. IV, we apply homogenization methods to calculate the effective nonlinear moving interface dynamics, first for explicit cases with variable coefficient permeability corresponding to horizontally and vertically layered media, then turning to the case of a doubly periodic permeability variation. In Sec. V, under the assumption of a thin gravity current, we derive and analyze using homogenized averaging a scalar nonlinear variable coefficient partial differential equation for this limit. We homogenize this equation and compute the leading-order and the corrected height fields. In Sec. VI, through a direct comparison between computational simulations of this equation and the homogenized dynamics, we provide compelling evidence for the success of the averaging approach for this nonlinear moving boundary problem.

\section{THE NONLINEAR INTERFACE PROBLEM}

The problem that motivates this study involves three fluid phases: a heterogeneous porous medium, and fluid composition effects that determine the density of the fluid and the interfacial tension between binary groupings of phases. The physics of this situation are complicated and involve issues such as capillary trapping and hysteretic constitutive relations. ${ }^{1}$ The modeling of such a system in a physically realistic way requires a compositional, multiphase model in which the composition of each fluid phase (water, DNAPL, and gas) and the volumetric phase distributions are evolved in time in a heterogeneous porous medium. In this work, we wish to analyze a simpler problem as a first step to give general guidance to assess the behavior of such systems and estimate the relative time scale for brine slumping. As such, we will ignore the multiphase and compositional nature of the motivating problem and focus on an analysis of the dynamics of a single-phase free-surface problem for a single fluid in a heterogeneous porous medium.

The situation we consider here is that of an initial volume of fluid in a porous media that is released and slumps under the force of gravity. In this context we refer to the volume as the volume of bulk porous medium that can be saturated by the volume of water in the initial region, assuming a constant porosity and field capacity. Experimentally, for example, this could represent crudely the flow of a core of liquid initially held in a vertical cylinder and then released into a porous media drained to field capacity. Alternatively, the fluid could represent the elevated brine layer injected into a water-saturated porous media, which does not quickly mix with the water, but forms an elevated slumping gravity current. Of course, the fully coupled water-brine system is more complex, requiring at the very least an evolving density variable, we focus upon the simpler single-fluid case for ease of exposition.

Flow in porous media is typically characterized by slow pressure-driven motion. In particular, for incompressible flow in a porous media without mass sources or sinks we have

$$
\begin{aligned}
& \mathbf{u}=-K(x, z)[\boldsymbol{\nabla} p+\rho g \hat{k}], \\
& \boldsymbol{\nabla} \cdot \mathbf{u}=0,
\end{aligned}
$$

where $\mathbf{u}$ is the fluid velocity, $p$ is the pressure, $K=k / \mu$, where $k$ is the permeability and $\mu$ is the viscosity, $\rho$ is the density of the fluid, $g$ is gravity, and $\hat{k}$ is a unit vector in the vertical direction. The quantity $K_{c} \equiv k \rho g / \mu$ is called the hydraulic conductivity, ${ }^{4}$ and we are interested in cases where this has a prescribed spatial variation. While Eqs. (1) and (2) are linear, the problem under consideration here is nonlinear due to the moving interface.

The interface defines the shape of the fluid mass as it flows through the medium. Mass conservation requires that $\mathbf{u} \cdot \hat{n}=\mathbf{U}_{I} \cdot \hat{n}$ at the interface, where $\mathbf{U}_{I}$ is the velocity of the interface and $\hat{n}$ is a unit normal vector. In the case of interest here, we represent the interface position by $z=h(x, t)$, where we assume symmetry about $x=0$ and represent the velocity of the interface as $\mathbf{U}_{I}=h_{t} \hat{k}$. Then, with the fluid velocity defined with components $\mathbf{u}=(u, w)$, this kinematic condition is written as

$$
\frac{\partial h}{\partial t}+u \frac{\partial h}{\partial x}=w, \quad \text { at } z=h .
$$

Additional conditions on the field variables are

$$
\begin{aligned}
& w=0, \quad \text { at } z=0, \\
& p=p_{A}, \quad \text { at } z=h,
\end{aligned}
$$

where $p_{A}$ is the atmospheric pressure. The first of these represents an impermeable layer at $z=0$ and the second states that the pressure at the free surface is atmospheric pressure (we can set $p_{A}=0$ without a loss of generality). The free surface condition (3) also requires boundary conditions, and these are given by 


$$
\begin{aligned}
& \frac{\partial h}{\partial x}=0, \quad \text { at } x=0, \\
& h=0, \quad \text { at } x=R(t),
\end{aligned}
$$

where $R$ is the radial position of the contact line. The first of these is a symmetry condition and the second is the condition of contact. A final condition states that the contact line position moves with the horizontal fluid velocity at the contact line boundary,

$$
\frac{d R}{d t}=u(R, t), \quad \text { at } x=R(t) .
$$

This condition can be derived from Eq. (3) with the use of conditions (4) and (7) and therefore is in some sense redundant. It does offer a clear physical picture of the motion of the contact line, however, and as a reduced form of Eq. (3) at the contact line it will appear again in one of our numerical approaches implemented in Sec. VI. The bulk of the analysis presented here will focus on Eqs. (1)-(3). A further discussion of the conditions (6)-(8) as well as alternative conditions useful for a numerical solution is presented in Secs. V and VI.

For initial conditions, we assume a given profile, $h(x, 0)=h_{0}(x)$, and we assume that initially that the fluid itself possesses no motion. Note that these equations form a coupled system, in which the boundary is coupled to the field variables through the moving interface condition. In Sec. V, we identify a slender asymptotic limit in which the interface position $h$ may be decoupled to yield a scalar, nonlinear, variable-coefficient partial differential equation. In general, the above system requires numerical inversion to handle the variable coefficient nature of the problem. However, in the next section, we present scaling arguments that connect to known similarity solutions and to the slender geometry in the case of a constant-coefficient permeability.

The above governing equations can be recast in terms of the field variable $\phi=p /(\rho g)+z$ by combining Eqs. (1) and (2) to obtain a variable coefficient elliptic problem,

$$
\boldsymbol{\nabla} \cdot\left[K_{c}(x, z) \boldsymbol{\nabla} \phi\right]=0,
$$

subject to boundary conditions

$$
\begin{aligned}
& \frac{\partial \phi}{\partial z}=0, \quad \text { at } z=0, \\
& \phi=h(x, t), \quad \text { at } z=h(x, t) .
\end{aligned}
$$

The kinematic boundary condition may also be recast in terms of the variable $\phi$ as

$$
\frac{\partial h}{\partial t}=\frac{\partial}{\partial x} \int_{0}^{h} K_{c} \frac{\partial \phi}{\partial x} d z,
$$

which is subject to the same boundary conditions as given above in Eqs. (6)-(8).

\section{SCALING ARGUMENTS FOR A GRAVITY CURRENT IN A POROUS MEDIA}

In this section we outline scaling arguments used to identify approximately how the fluid in the porous medium flows under the force of gravity (as governed by the above equations). Here we assume that the permeability coefficient is constant. These scaling arguments lead to the identification of simple formulas for the spatial evolution of the fluid mass as well as similarity variables for which an exact (selfsimilar) solution exists. This self-similar form is well known. ${ }^{4,5}$ Similar analyses have been performed by Huppert ${ }^{11}$ for viscous gravity currents, Huppert and Woods ${ }^{12}$ for gravity currents in porous layers, and by Acton et al. ${ }^{13}$ for viscous gravity currents over porous media. Our purpose for outlining the results here is for a later comparison and use of them in the context of porous media flows with nonuniform permeability.

In component form, the two-dimensional equations are

$$
\begin{aligned}
& u=-K \frac{\partial p}{\partial x}, \\
& w=-K\left(\frac{\partial p}{\partial z}+\rho g\right), \\
& \frac{\partial u}{\partial x}+\frac{\partial w}{\partial z}=0,
\end{aligned}
$$

and

$$
\frac{\partial h}{\partial t}+u \frac{\partial h}{\partial x}=w, \quad \text { at } z=h .
$$

We let $H$ and $R$ represent vertical and horizontal length scales associated with the shape of the fluid mass and $T$ represent the time scale. The pressure and two velocity scales $P, U$, and $W$ will be determined in terms of the physical parameters and the space and time scales. Additionally, we shall identify how $H$ and $R$ scale with time.

In determining the balances that follow, we shall assume that in the continuity equation (15) both terms balance and that in the kinematic condition (16) all terms balance. Two different limits that we consider will arise from different balances that are possible with respect to the vertical component of Darcy's equation (14).

A balance of terms in the continuity equation (15) and also in the kinematic condition (16) implies that

$$
W \sim \frac{H}{R} U, \quad \frac{H}{T} \sim \frac{U H}{R} \sim W .
$$

It follows from this that

$$
U \sim \frac{R}{T}, \quad W \sim \frac{H}{T} .
$$

The horizontal component of Darcy's equation (13) implies that

$$
U \sim \frac{K P}{R} .
$$

Balances (18) and (19) then give the pressure scale as

$$
P \sim \frac{R^{2}}{K T} .
$$


The terms in the vertical component of Darcy's equation (14) have the associated scales $U H / R, K P / H$, and $K \rho g$, respectively. In light of the above results, these are

$$
\frac{H K P}{R^{2}}, \quad \frac{K P}{H}, \quad K \rho g
$$

In what follows we consider two limits with respect to the length scales $H$ and $R$, which lead to two different possible balances with respect to this equation.

If $H \ll R$, then the terms in (21) suggest that only the second two balance (the first term is smaller by a factor of $H^{2} / R^{2}$ ), which represents a physical balance between the vertical pressure gradient and gravity; a hydrostatic balance. In this case, we obtain

$$
P \sim \rho g H .
$$

A comparison of (20) and (22) gives

$$
\frac{R^{2}}{K T} \sim \rho g H
$$

Finally, we relate the length scales $H$ and $R$ through a fixed volume (per unit length)

$$
V_{L} \sim R H,
$$

to arrive at a scaling dynamics for $R$ given by

$$
R \sim\left(\rho g K V_{L} T\right)^{1 / 3} .
$$

This is the scaling for which the liquid blob spreads in the horizontal direction when $H \ll R$. We can determine the scaling for $H$, using $V_{L} \sim R H$,

$$
H \sim \frac{V_{L}}{\left(\rho g K V_{L} T\right)^{1 / 3}} .
$$

The notion of a time scale for problems of this nature is a bit of an elusive concept. However, since we have assumed that $H \ll R$, comparing balances (25) and (26) suggests a time scale upon which the balance is appropriate, namely

$$
T \gg \frac{V_{L}^{1 / 2}}{\rho g K} .
$$

One interpretation of this is as a relaxation time scale to this thin $(H \ll R)$ configuration.

An alternative balance is possible at earlier times if the horizontal scales and vertical scales balance. That is, if $H$ $\sim R$, then (21) suggests a pressure scale in which all three terms balance given by

$$
P \sim \rho g R,
$$

which in conjunction with balance (20) gives

$$
R \sim \rho g K T .
$$

The volume constraint $V_{L} \sim H R$ suggests that

$$
H \sim \frac{V_{L}}{\rho g K T} .
$$

Since we have assumed that $H \sim R$, balances (29) and (30) suggest a time scale on which the balance is appropriate, namely

$$
T \sim \frac{V_{L}^{1 / 2}}{\rho g K} .
$$

\section{A. Similarity solution based on scaling for $H \ll R$}

We now focus in more detail on the limit $H \ll R$ described in the previous section. In this limit, the leadingorder equations are

$$
\begin{aligned}
& u=-K \frac{\partial p}{\partial x}, \\
& 0=-K\left(\frac{\partial p}{\partial z}+\rho g\right), \\
& \frac{\partial u}{\partial x}+\frac{\partial w}{\partial z}=0,
\end{aligned}
$$

and

$$
\frac{\partial h}{\partial t}+u \frac{\partial h}{\partial x}=w, \quad \text { on } \quad z=h(x, t) .
$$

Recalling the dimensional form for the vertical component of Darcy's law given by (14) and the scaling for each term given by (21), we note that in the limit where $H \ll R$ the left-hand side of (14) can be neglected.

The constant volume per unit length $V_{L}$ is

$$
V_{L}=2 \int_{0}^{R(t)} h(x, t) d x,
$$

where $R(t)$ is the location of the contact line where $h[R(t), t]=0$. In the following analysis we derive an evolution equation for the shape $h(x, t)$ of the liquid volume, and then apply the scalings of the previous section to formulate a similarity solution representation for the shape.

Equation (33) implies that the pressure is hydrostatic to leading order. With $p(z=h)=0$ this implies

$$
p=\rho g[h(x, t)-z] .
$$

It follows that the horizontal velocity is given by

$$
u=-K_{c} \frac{\partial h}{\partial x}
$$

The continuity equation then gives the vertical velocity

$$
w=K_{c}\left[\frac{\partial^{2} h}{\partial x^{2}}\right] z .
$$

Substituting the results for $u$ and $w$ into the kinematic boundary condition (35) leads to an evolution equation for the shape

$$
\frac{\partial h}{\partial t}=K_{c} \frac{\partial}{\partial x}\left(h \frac{\partial h}{\partial x}\right) .
$$

Recall that we have assumed that $K_{c}$ is constant in this derivation. 
We summarize the known similarity solutions derived by Barenblatt $^{5}$ for this scalar nonlinear PDE. Based on the scalings described in the previous section, we introduce a similarity variable,

$$
\eta=\frac{x}{\left(K_{c} V_{L} t\right)^{1 / 3}},
$$

and a rescaled height function $f(\eta)$, where

$$
h=\frac{V_{L}}{\left(K_{c} V_{L} t\right)^{1 / 3}} f(\eta)
$$

This solution can be expressed as

$$
h(x, t)=\frac{V_{L}}{6\left(K_{c} V_{L} t\right)^{1 / 3}}\left[\left(\frac{9}{2}\right)^{2 / 3}-\frac{x^{2}}{\left(K_{c} V_{L} t\right)^{2 / 3}}\right],
$$

where

$$
h_{\max }(t)=\frac{1}{6}\left(\frac{9}{2}\right)^{2 / 3} \frac{V_{L}}{\left(K_{c} V_{L} t\right)^{1 / 3}},
$$

and

$$
R(t)=\left(\frac{9}{2}\right)^{1 / 3}\left(K_{c} V_{L} t\right)^{1 / 3} .
$$

The above three formulas apply when $h_{\max }(t) \ll R(t)$ or equivalently when

$$
t \gg \frac{\sqrt{V_{L}}}{4 \sqrt{3} K_{c}} .
$$

We will make contact with the similarity solution presented here for the constant permeability porous medium using methods of homogenized averaging applied to the more general case of a variable permeability medium below. In particular, in Sec. IV we shall show how the above similarity solution can be applied to describe the averaged motion of a gravity current through porous media with vertically and/or horizontally layered permeability variations. Also, in Sec. V, we will make use of slender asymptotics applied to the problem defined in Sec. II to decouple the height field from the elliptic operator. This leads to a scalar nonlinear PDE with variable coefficients (i.e., $K_{c}$ not uniform in space) that will be analyzed using homogenization methods and connected back to the similarity solution presented here.

First, however, we discuss the basic time scales implied by these solutions in maintaining a localized, elevated axisymmetric density field.

\section{B. Similarity solution in axisymmetric coordinates}

If we follow a scaling analysis similar to that presented in the previous section for rectangular coordinates, we find that the limit $H \ll R$ in the axisymmetric case has

$$
\begin{aligned}
& R \sim\left(K_{c} V T\right)^{1 / 4}, \\
& H \sim \frac{V}{\left(K_{c} V T\right)^{1 / 2}},
\end{aligned}
$$

when $T \gg V^{1 / 3} / K_{c}$, where the volume is given by

$$
V=2 \pi \int_{0}^{R(t)} r h(r, t) d r .
$$

We can obtain a similarity solution in the limit $H \ll R$ in terms of the similarity variable,

$$
\eta=\frac{r}{\left(K_{c} V t\right)^{1 / 4}},
$$

and a rescaled height function $f(\eta)$, where

$$
h=\frac{V}{\left(K_{c} V t\right)^{1 / 2}} f(\eta) .
$$

This solution can be expressed as

$$
h(r, t)=\frac{V}{\left(K_{c} V t\right)^{1 / 2}}\left(\frac{1}{2 \sqrt{\pi}}-\frac{1}{8} \frac{r^{2}}{\left(K_{c} V t\right)^{1 / 2}}\right),
$$

where

$$
h_{\max }(t)=\frac{1}{2 \sqrt{\pi}} \frac{V}{\left(K_{c} V t\right)^{1 / 2}},
$$

and

$$
R(t)=\frac{2}{\pi^{1 / 4}}\left(K_{c} V t\right)^{1 / 4} .
$$

Again, the time regime represented here is set by the condition $h_{\max }(t) \ll R(t)$, which gives

$$
t \gg \frac{1}{\left(r^{4 / 3} \pi^{1 / 3}\right)} \frac{V^{1 / 3}}{K_{c}},
$$

and can be compared to Eq. (27).

\section{Predictions}

Practically, when pumping brine into a field to assist in the cleanup process, it is important to assess how much brine must be continually injected to maintain an elevated, and spatially localized brine layer. We may use the similarity solutions derived above, for the practical case of a radial distribution, to assess basic leakage time scales in a porous medium for the slumping gravity current in the absence of pumping. This pure relaxation analysis gives a good description of how long the elevated density layer may persist in a field cleanup. Of course, with similarity solutions, initially the similarity solutions diverge, and we first must overcome this problem by waiting a transient time scale to allow the similarity solution to develop, and then we may use the ensuing evolution to assess the slumping time scale. We approach this transient time scale by matching a given distribution of fluid, integrating backward in time following the similarity scalings to assess an initial volume and a transient time scale that would have passed to reach the current state as a means to eliminate the transient time scale.

Consider a mass of fluid of parabolic shape whose height is $h^{*}$ and whose radius is $R^{*}$. We are interested in the evolution of this volume of fluid under the force of gravity. In order to make use of our similarity solution described above, 
TABLE I. Fractional height of fluid mass, time in days, and fraction loss of volume outside given radius: The predictions use $h^{*}=10 \mathrm{~m}, R^{*}=34.4 \mathrm{~m}$, and $K_{c}=10^{-4} \mathrm{~m} / \mathrm{s}$. The corresponding value of $t^{*}$ given by Eq. (56) is 1.72 days.

\begin{tabular}{ccc}
\hline \hline$\lambda=h / h^{*}$ & time in days & $\omega=V_{\text {out }} / V$ \\
\hline 1 & 0 & 0 \\
0.8 & 0.97 & 0.04 \\
0.5 & 5.1 & 0.25 \\
0.25 & 25.7 & 0.56 \\
0.1 & 170 & 0.81 \\
0 & $\infty$ & 1 \\
\hline \hline
\end{tabular}

we can associate this shape with a similarity solution of some initial volume at a particular time $t^{*}$. In terms of $h^{*}$ and $R^{*}$, the volume and associated time are given by

$$
V=\frac{\pi}{2} h^{*}\left(R^{*}\right)^{2}, \quad t^{*}=\frac{\left(R^{*}\right)^{2}}{8 K_{c} h^{*}} .
$$

These relationships follow from Eqs. (53) and (54).

Now, if we are interested in predicting the time it takes for this mass of fluid to evolve from its initial height $h^{*}$ to some other height $h(t)$, where we define $\lambda=h(t) / h^{*}$, we find that

$$
\lambda=\frac{V^{1 / 2}}{2 h^{*} \sqrt{\pi K_{c}\left(t^{*}+\widetilde{t}\right)}}=\sqrt{\frac{t^{*}}{t^{*}+\widetilde{t}}},
$$

where we have introduced the time $\tilde{t}$ that measures the time of evolution from the shape with height $h^{*}$ and radius $R^{*}$. We solve the above equation for $\tilde{t}$ and find that

$$
\tilde{t}=t^{*}\left(\frac{1}{\lambda^{2}}-1\right)=\left(\frac{\left(R^{*}\right)^{2}}{8 K_{c} h^{*}}\right)\left(\frac{1}{\lambda^{2}}-1\right) .
$$

Table I shows some data representative of an idealized example of groundwater flow. For $t<t^{*}$, no fluid is outside the radius $R^{*}$. For $t>t^{*}$, the volume of fluid $V_{\text {out }}$ outside this boundary can be represented by the integral

$$
V_{\text {out }}(t)=2 \pi \int_{R^{*}}^{R_{0}(t)} r h(r, t) d r,
$$

where $h(r, t)$ is given by Eq. (52). Upon working out this integral, we find that

$$
\omega=\frac{V_{\text {out }}(t)}{V}=\left[1-\left(\frac{\pi^{1 / 4} R^{*}}{2\left[K_{c} V\left(t^{*}+\widetilde{t}\right)\right]^{1 / 4}}\right)^{2}\right]^{2} .
$$

In a similar manner to the above calculation, we find that the time for which a certain percentage of the volume of fluid (measured by $\omega$ ) to be outside a radius $R^{*}$ is given by

$$
\tilde{t}=t^{*}\left(\frac{1}{(1-\sqrt{\omega})^{2}}-1\right)=\left(\frac{\left(R^{*}\right)^{2}}{8 K_{c} h^{*}}\right)\left(\frac{1}{(1-\sqrt{\omega})^{2}}-1\right) .
$$

Perhaps more simply, we can relate the fractional height remaining $\lambda$ to the fractional volume lost $\omega$ as

$$
\omega=(1-\lambda)^{2} \text {. }
$$

The corresponding values of $\omega$ are also shown in Table I. This is effectively just a geometrical relationship given that our similarity shape is a parabola.

\section{EFFECTIVE FRONT DYNAMICS: HOMOGENIZED AVERAGING OF THE GRAVITY CURRENT}

With the similarity scalings and solutions identified, we proceed to the more complex case involving a nonconstant coefficient permeability. In this section we apply methods of homogenized averaging to assess the effect of the variable permeability upon the slumping interface. We note that there is a tremendous literature for homogenization in many contexts, ${ }^{14-21}$ most typically applied to linear problems. Here, we stress the problem addressed in this paper is nonlinear through the moving boundary condition. As such, we show formally how the averaging procedures work in this more difficult context, and document its success numerically below in Sec. VI.

To leading order, we will find that the homogenized equations share essentially the same form as the constant coefficient equations analyzed previously, with the constant permeabilities replaced by constant, effective permeabilities. As such, the time-scale analyses from the previous section will apply to these averaged dynamics in an identical fashion, only with the permeability replaced by an effective permeability. We will document how this averaging works in this nonlinear problem in cases with horizontally or vertically layered permeabilities. We also describe a general case in which the permeability varies in two spatial directions. Here we will present only the leading-order calculations, and defer the interesting corrections to future work. In what follows, the isotropic form of the permeability is modified through the averaging procedure to different permeabilities in the horizontal and vertical directions. We show how to make contact to the similarity scalings derived in Sec. III through the leading-order homogenization.

\section{A. Homogenization theory: Vertical layers}

In this section we consider the situation in which the permeability of the porous medium is rapidly varying in the horizontal direction so that layers of constant permeability are oriented vertically. Specifically we write $K_{c}=K_{c}(x / \epsilon)$, where $\epsilon$ is a small parameter; we shall examine the limit $\epsilon \rightarrow 0$. For simplicity, we shall assume that $K_{c}(x / \epsilon)$ is a periodic function with unit period. We consider the governing equations in the two-dimensional setting,

$$
\begin{aligned}
& \mathbf{u}=-K_{c}(X) \boldsymbol{\nabla} \phi, \\
& \boldsymbol{\nabla} \cdot \mathbf{u}=0,
\end{aligned}
$$

where $X=x / \epsilon$. The kinematic boundary condition is given by Eq. (3).

We proceed using a multiscale analysis in which $x$ and $X$ are treated as independent variables so that

$$
\frac{\partial}{\partial x} \rightarrow \frac{\partial}{\partial x}+\frac{1}{\epsilon} \frac{\partial}{\partial X}
$$

Further, we expand the variables 


$$
\begin{aligned}
& \phi(x, X, z, t)=\phi_{0}(x, X, z, t)+\epsilon \phi_{1}(x, X, z, t)+\cdots, \\
& u(x, X, z, t)=u_{0}(x, X, z, t)+\epsilon u_{1}(x, X, z, t)+\cdots, \\
& w(x, X, z, t)=w_{0}(x, X, z, t)+\epsilon w_{1}(x, X, z, t)+\cdots, \\
& h(x, X, t)=h_{0}(x, X, t)+\epsilon h_{1}(x, X, t)+\cdots .
\end{aligned}
$$

At $\mathcal{O}(1 / \epsilon)$ the horizontal component of Darcy's equation gives

$$
0=K_{c}(X) \frac{\partial \phi_{0}}{\partial X}
$$

which leads to the conclusion that $\phi_{0}$ is independent of $X$ so $\phi_{0}=\phi(x, z, t)$. At $\mathcal{O}(1)$ we find expressions for the leadingorder velocity components,

$$
\begin{aligned}
& u_{0}(x, X, z, t)=-K_{c}(X)\left[\frac{\partial \phi_{0}}{\partial x}+\frac{\partial \phi_{1}}{\partial X}\right], \\
& w_{0}(x, X, z, t)=-K_{c}(X) \frac{\partial \phi_{0}}{\partial z} .
\end{aligned}
$$

Since $\phi_{0}$ is independent of $Z$ we can average $w_{0}$ to find that

$$
\left\langle w_{0}\right\rangle=-\left\langle K_{c}\right\rangle \frac{\partial \phi_{0}}{\partial z} .
$$

Here $\langle\cdot\rangle=\int_{0}^{-1} \cdot d X$. In order to work out the average of $u_{0}$, we shall first examine the equation for $\phi$,

$$
0=\boldsymbol{\nabla} \cdot\left[K_{c}(X) \boldsymbol{\nabla} \phi\right] .
$$

Expanding Eq. (74) gives at $\mathcal{O}\left(1 / \epsilon^{2}\right)$ that

$$
0=\frac{\partial}{\partial X}\left[K_{c}(X) \frac{\partial \phi_{0}}{\partial X}\right],
$$

which we know is identically satisfied since $\phi_{0}$ is independent of $X$. At $\mathcal{O}(1 / \epsilon)$ we find that

$$
\begin{aligned}
\frac{\partial}{\partial X}\left[K_{c}(X) \frac{\partial \phi_{1}}{\partial X}\right]= & -\frac{\partial}{\partial x}\left[K_{c}(X) \frac{\partial \phi_{0}}{\partial X}\right] \\
& -\frac{\partial}{\partial X}\left[K_{c}(X) \frac{\partial \phi_{0}}{\partial x}\right],
\end{aligned}
$$

which upon integrating once gives

$$
K_{c}(X)\left[\frac{\partial \phi_{1}}{\partial X}+\frac{\partial \phi_{0}}{\partial x}\right]=A(x, z, t),
$$

where $A(x, z, t)$ is an integration constant to be determined. Averaging this result and assuming that $\phi_{1}$ is periodic [that is, $\left.\phi_{1}(X=0)=\phi_{1}(X=1)\right]$, we have

$$
A(x, z, t)=\left\langle\frac{1}{K_{c}}\right\rangle^{-1} \frac{\partial \phi_{0}}{\partial x} .
$$

Now Eq. (71) implies that $u_{0}(x, X, z, t)=-A(x, z, t)$ is independent of $X$, so

$$
u_{0}=\left\langle u_{0}\right\rangle=-\left\langle\frac{1}{K_{c}}\right\rangle^{-1} \frac{\partial \phi_{0}}{\partial x} .
$$

Expanding Eq. (74) gives, at $\mathcal{O}(1)$,

$$
\begin{aligned}
& \frac{\partial}{\partial X}\left[K_{c}(X)\left(\frac{\partial \phi_{1}}{\partial x}+\frac{\partial \phi_{2}}{\partial X}\right)\right] \\
& =-\frac{\partial}{\partial x}\left[K_{c}(X)\left(\frac{\partial \phi_{0}}{\partial x}+\frac{\partial \phi_{1}}{\partial X}\right)\right]-K_{c}(X) \frac{\partial^{2} \phi_{0}}{\partial z^{2}} .
\end{aligned}
$$

Averaging this equation and making use of Eqs. (77) and (78) leads to the result that

$$
0=\left\langle K_{c}\right\rangle \frac{\partial^{2} \phi_{0}}{\partial z^{2}}+\left\langle\frac{1}{K_{c}}\right\rangle^{-1} \frac{\partial^{2} \phi_{0}}{\partial x^{2}} .
$$

This equation shows the anisotropy that is built into the equations from the vertical layering, with one coefficient corresponding to the arithmetic mean of the permeabilities and the other corresponding to the harmonic mean as observed by others, in the absence of the nonlinear moving boundary condition. ${ }^{4,20}$ These coefficients are reversed relative to the horizontally layered media discussed in the next section.

Next, examining the continuity equation $\boldsymbol{\nabla} \cdot \mathbf{u}=0$ we find that the $\mathcal{O}(1 / \epsilon)$ problem is identically satisfied since $u_{0}$ $=u_{0}(x, z, t)$ and that the $\mathcal{O}(1)$ problem is

$$
\frac{\partial\left\langle u_{0}\right\rangle}{\partial x}+\frac{\partial\left\langle w_{0}\right\rangle}{\partial z}=0 .
$$

Finally, if we expand the boundary condition (3) applied at $z=h(x, X, t)$, we find that at $\mathcal{O}(1 / \epsilon)$,

$$
0=u_{0} \frac{\partial h_{0}}{\partial X},
$$

which implies that $h_{0}=h_{0}(x, t)$ is independent of $X$. The $\mathcal{O}(1)$ contribution from Eq. (3) is

$$
u_{0} \frac{\partial h_{1}}{\partial X}=-\frac{\partial h_{0}}{\partial t}-u_{0} \frac{\partial h_{0}}{\partial x}+w_{0} .
$$

Averaging this equation (integrate in $X$ from 0 to 1 ) and assuming the periodicity of $h_{1}$ gives

$$
\frac{\partial h_{0}}{\partial t}+\left\langle u_{0}\right\rangle \frac{\partial h_{0}}{\partial x}=\left\langle w_{0}\right\rangle .
$$

So the full set of averaged leading-order equations are

$$
\begin{aligned}
& \left\langle u_{0}\right\rangle=-\left\langle\frac{1}{K_{c}}\right\rangle^{-1} \frac{\partial \phi_{0}}{\partial x}, \\
& \left\langle w_{0}\right\rangle=-\left\langle K_{c}\right\rangle \frac{\partial \phi_{0}}{\partial z}, \\
& 0=\frac{\partial\left\langle u_{0}\right\rangle}{\partial x}+\frac{\partial\left\langle w_{0}\right\rangle}{\partial z},
\end{aligned}
$$

and

$$
\frac{\partial h_{0}}{\partial t}+\left\langle u_{0}\right\rangle \frac{\partial h_{0}}{\partial x}=\left\langle w_{0}\right\rangle .
$$

The average horizontal flow is proportional to the harmonic mean of the permeabilities, while the vertical flow is proportional to the arithmetic mean of the permeabilities. This is a well-known property of layered media ${ }^{4,20}$ and the difference can be significant, especially if the permeability of 
any layer is near zero. In such a case there would be vertical flow through the regions of higher permeability but nearly no horizontal flow, as the fluid must attempt to flow across the regions of low permeability.

\section{B. Similarity scalings for the averaged dynamics: Vertical layers}

To make contact to the similarity analyses presented in Sec. III, we now subject Eqs. (86)-(89) to the limit $H \ll R$. We have [in terms of the leading-order pressure $p_{0}$ $\left.=p_{0}(x, z, t)\right]$

$$
\begin{aligned}
& \left\langle u_{0}\right\rangle=-\left\langle\frac{1}{K}\right\rangle^{-1} \frac{\partial p_{0}}{\partial x}, \\
& 0=-\frac{\partial p_{0}}{\partial z}-\rho g, \\
& 0=\frac{\partial\left\langle u_{0}\right\rangle}{\partial x}+\frac{\partial\left\langle w_{0}\right\rangle}{\partial z},
\end{aligned}
$$

and

$$
\frac{\partial h_{0}}{\partial t}+\left\langle u_{0}\right\rangle \frac{\partial h_{0}}{\partial x}=\left\langle w_{0}\right\rangle .
$$

Note that only the effective permeability appearing in the horizontal component of Darcy's equation governs the slumping gravity current. The form of these equations is equivalent to Eqs. (32)-(35), from which we derived the similarity solution. Consequently, Eqs. (43)-(46) apply here simply by replacing $K_{c}$ in those formulas with $\left\langle 1 / K_{c}\right\rangle^{-1}$. We confirm in Sec. VI that these equations give rise to a leadingorder problem that agrees well with numerical simulations of the full PDE that incorporates a permeability function with dependence of the form $K_{c}(x / \epsilon)$.

\section{Homogenization theory: Horizontal layers}

In this section we consider the situation in which the permeability of the porous medium is rapidly varying in the vertical direction, that is, layers of constant permeability are oriented horizontally. Specifically we write $K_{c}=K_{c}(z / \epsilon)$, where $\epsilon$ measures the length scale of the variation and examine the limit $\epsilon \longrightarrow 0$. For simplicity, we shall assume that $K_{c}(z / \epsilon)$ is a periodic function with period unity. The governing equations are the same as in the case of vertical layers, except that Darcy's equation is now written as

$$
\mathbf{u}=-K_{c}(Z) \nabla \phi,
$$

where $Z=z / \epsilon$.

We proceed using a multiscale analysis in which $z$ and $Z$ are treated as independent variables, so that

$$
\frac{\partial}{\partial z} \rightarrow \frac{\partial}{\partial z}+\frac{1}{\epsilon} \frac{\partial}{\partial Z}
$$

Further, we expand the variables

$$
\begin{aligned}
& \phi(x, z, Z, t)=\phi_{0}(x, z, Z, t)+\epsilon \phi_{1}(x, z, Z, t)+\cdots, \\
& u(x, z, Z, t)=u_{0}(x, z, Z, t)+\epsilon u_{1}(x, z, Z, t)+\cdots,
\end{aligned}
$$

$$
\begin{aligned}
& w(x, z, Z, t)=w_{0}(x, z, Z, t)+\epsilon w_{1}(x, z, Z, t)+\cdots, \\
& h(x, t)=h_{0}(x, t)+\epsilon h_{1}(x, t)+\cdots
\end{aligned}
$$

Note here that $h$ is assumed to not depend on the fast scale $Z$. At $\mathcal{O}(1 / \epsilon)$ the vertical component of Darcy's equation is

$$
0=K_{c}(Z) \frac{\partial \phi_{0}}{\partial Z}
$$

which leads to the conclusion that $\phi_{0}$ is independent of $Z$ so $\phi_{0}=\phi(x, z, t)$. At $\mathcal{O}(1)$ we find expressions for the leadingorder velocity components,

$$
\begin{aligned}
& u_{0}(x, z, Z, t)=-K_{c}(Z) \frac{\partial \phi_{0}}{\partial x}, \\
& w_{0}(x, z, Z, t)=-K_{c}(Z)\left[\frac{\partial \phi_{0}}{\partial z}+\frac{\partial \phi_{1}}{\partial Z}\right] .
\end{aligned}
$$

Since $\phi_{0}$ is independent of $Z$ we can average $u_{0}$ to find that

$$
\left\langle u_{0}\right\rangle=-\left\langle K_{c}\right\rangle \frac{\partial \phi_{0}}{\partial x} .
$$

Here $\langle\cdot\rangle=\int_{0}^{1} \cdot d Z$. In order to work out the average of $w_{0}$, we shall first examine the equation for $\phi$,

$$
0=\boldsymbol{\nabla} \cdot\left[K_{c}(Z) \boldsymbol{\nabla} \phi\right] .
$$

Expanding Eq. (104) gives at $\mathcal{O}\left(1 / \epsilon^{2}\right)$ that

$$
0=\frac{\partial}{\partial Z}\left[K_{c}(Z) \frac{\partial \phi_{0}}{\partial Z}\right],
$$

which we know is identically satisfied since $\phi_{0}$ is independent of $Z$. At $\mathcal{O}(1 / \epsilon)$ we find that

$$
\begin{aligned}
\frac{\partial}{\partial Z}\left[K_{c}(Z) \frac{\partial \phi_{1}}{\partial Z}\right]= & -\frac{\partial}{\partial z}\left[K_{c}(Z) \frac{\partial \phi_{0}}{\partial Z}\right] \\
& -\frac{\partial}{\partial Z}\left[K_{c}(Z) \frac{\partial \phi_{0}}{\partial z}\right],
\end{aligned}
$$

which upon integrating once gives

$$
K_{c}(Z)\left[\frac{\partial \phi_{1}}{\partial Z}+\frac{\partial \phi_{0}}{\partial z}\right]=A(x, z, t),
$$

where $A(x, z, t)$ is an integration constant to be determined. Averaging this result and assuming that $\phi_{1}$ is periodic in $Z$, we have

$$
A(x, z, t)=\left\langle\frac{1}{K_{c}}\right\rangle^{-1} \frac{\partial \phi_{0}}{\partial z} .
$$

Now Eq. (102) implies that $w_{0}(x, z, Z, t)=-A(x, z, t)$ is independent of $Z$ using Eq. (107). Therefore, using (108) the averaged $w_{0}$ is

$$
\left\langle w_{0}\right\rangle=-\left\langle\frac{1}{K_{c}}\right\rangle^{-1} \frac{\partial \phi_{0}}{\partial z} .
$$

Continuing to the $\mathcal{O}(1)$ problem from Eq. (104) gives 


$$
\begin{aligned}
\frac{\partial}{\partial Z}\left[K_{c}(Z) \frac{\partial \phi_{2}}{\partial Z}\right]= & -\frac{\partial}{\partial x}\left[K_{c}(Z) \frac{\partial \phi_{0}}{\partial x}\right] \\
& -\frac{\partial}{\partial z}\left[K_{c}(Z)\left(\frac{\partial \phi_{1}}{\partial Z}+\frac{\partial \phi_{0}}{\partial z}\right)\right] \\
& -\frac{\partial}{\partial Z}\left[K_{c}(Z) \frac{\partial \phi_{1}}{\partial z}\right] .
\end{aligned}
$$

Integrating this equation once, using the result for $A(x, z, t)$ and periodicity of $\phi_{2}$, we find that

$$
0=\left\langle K_{c}\right\rangle \frac{\partial^{2} \phi_{0}}{\partial x^{2}}+\left\langle\frac{1}{K_{c}}\right\rangle^{-1} \frac{\partial^{2} \phi_{0}}{\partial z^{2}} .
$$

Here the same two averages of the permeability function appear but are associated with the opposite terms as compared to the case of vertical layers.

Next, examining the continuity equation $\boldsymbol{\nabla} \cdot \mathbf{u}=0$ we find that the $\mathcal{O}(1 / \epsilon)$ problem is identically satisfied since $w_{0}$ $=w_{0}(x, z, t)$ and that the averaged $\mathcal{O}(1)$ problem is

$$
\frac{\partial\left\langle u_{0}\right\rangle}{\partial x}+\frac{\partial\left\langle w_{0}\right\rangle}{\partial z}=0 .
$$

Finally, we must address the boundary condition (3) applied at $z=h(x, t)$. We note that the coefficients $u_{0}(x, z, Z, t)$ and $w_{0}(x, z, t)$ must be evaluated at $z=h_{0}(x, t)$. It is clear what to do in the case of $w_{0}$, which does not depend on the fast scale $Z$. However, $u_{0}$ does depend on $Z$ and needs to be evaluated at $z=h_{0}$. In order to get an equation for $h_{0}$ that approximates the full problem, we shall formally average the boundary condition in $Z$ as if it were an equation that applied in a region surrounding the actual boundary and then evaluate the result at $z=h_{0}(x, t)$. Doing so gives the prediction

$$
\frac{\partial h_{0}}{\partial t}+\left\langle u_{0}\right\rangle \frac{\partial h_{0}}{\partial x}=\left\langle w_{0}\right\rangle
$$

Justification in this approach is given in the slender limit case in which we show that the full numerical solution compares favorably with this approximation. Further justification of this result can be obtained by parametrizing the interface by its horizontal position $x=L(z, t)$ rather than its height. We would then take $L(z, Z, t)$ to be a function of the fast variable and identify a mathematical problem essentially like that considered above for the case of vertically oriented layers. We show in Appendix A that, under suitable assumptions, doing so leads to an equation identical to (113).

With this in mind the full set of averaged leading-order equations are

$$
\begin{aligned}
& \left\langle u_{0}\right\rangle=-\left\langle K_{c}\right\rangle \frac{\partial \phi_{0}}{\partial x}, \\
& \left\langle w_{0}\right\rangle=-\left\langle\frac{1}{K_{c}}\right\rangle^{-1} \frac{\partial \phi_{0}}{\partial z}, \\
& 0=\frac{\partial\left\langle u_{0}\right\rangle}{\partial x}+\frac{\partial\left\langle w_{0}\right\rangle}{\partial z},
\end{aligned}
$$

and

$$
\frac{\partial h_{0}}{\partial t}+\left\langle u_{0}\right\rangle \frac{\partial h_{0}}{\partial x}=\left\langle w_{0}\right\rangle .
$$

Compare these with Eqs. (86)-(89). Notice that here the average horizontal flow is proportional to the arithmetic mean of the permeabilities, while the vertical flow is proportional to the harmonic mean of the permeabilities.

\section{Similarly scalings for the averaged dynamics: Horizontal layers}

To make contact to the similarity analyses presented in Sec. III, we now subject Eqs. (114)-(117) to the limit $H$ $\ll R$. We have [in terms of the leading-order pressure $p_{0}$ $\left.=p_{0}(x, z, t)\right]$

$$
\begin{aligned}
& \left\langle u_{0}\right\rangle=-\langle K\rangle \frac{\partial p_{0}}{\partial x}, \\
& 0=-\frac{\partial p_{0}}{\partial z}-\rho g, \\
& 0=\frac{\partial\left\langle u_{0}\right\rangle}{\partial x}+\frac{\partial\left\langle w_{0}\right\rangle}{\partial z},
\end{aligned}
$$

and

$$
\frac{\partial h_{0}}{\partial t}+\left\langle u_{0}\right\rangle \frac{\partial h_{0}}{\partial x}=\left\langle w_{0}\right\rangle .
$$

Note again that only the effective permeability appearing in the horizontal component of Darcy's equation governs the slumping gravity current. The form of these equations is equivalent to Eqs. (32)-(35) from which we derived the similarity solution. Consequently, Eqs. (43)-(46) apply here simply by replacing $K_{c}$ with $\left\langle K_{c}\right\rangle$. We confirm in Sec. VI that these equations give rise to a leading-order problem that agrees well with numerical simulations of the full PDE that incorporates a permeability function with dependence of the form $K_{c}(z / \epsilon)$. We note that there is a length scale set by the permeability variations in the vertical direction and also one set by the thinness of the fluid layer and when implementing specific permeability functions $K_{c}(z / \epsilon)$ one must keep in mind the relative size of these two length scales.

\section{E. Homogenization theory: Two-dimensional periodicity}

In this section we describe the leading-order homogenization problem for a case in which there is rapid variation of the permeability function in two directions so that $K_{c}(X, Z)$, where $X=x / \epsilon$ and $Z=z / \epsilon$. For simplicity we assume that this function is periodic in $X$ and $Z$ with unit period in each direction. This case is important because most natural porous medium systems have spatial variability in multiple dimensions, making this a useful extension to our analysis.

We focus on the problem in terms of the function $\phi$, which satisfies

$$
\boldsymbol{\nabla} \cdot\left[K_{c}(X, Z) \boldsymbol{\nabla} \phi\right]=0,
$$

subject to the free-surface boundary condition 


$$
\frac{\partial h}{\partial t}+u \frac{\partial h}{\partial x}=w, \quad \text { at } z=h .
$$

In order to pursue a homogenization with two fast variables $X$ and $Z$, we need to decide how to handle the application of the boundary condition at $z=h$. We discuss this below after revisiting the approach used for the equations applied in the bulk. The standard homogenization techniques for bulk quantities such as $\phi$ proceed by assuming that $\phi$ $=\phi(x, z, X, Z)$ depends on four independent variables (for ease of notation we suppress dependence on time in this list). We shall outline the known steps and results for the homogenization of Eq. (122).

We assume an expansion for $\phi$ of the form

$$
\phi=\phi_{0}(x, z, X, Z)+\epsilon \phi_{1}(x, z, X, Z)+\cdots .
$$

Equation (122) is expanded as

$$
\begin{aligned}
\left(\frac{\partial}{\partial x}\right. & \left.+\frac{1}{\epsilon} \frac{\partial}{\partial X}\right)\left[K_{c}(X, Z)\left(\frac{\partial \phi}{\partial x}+\frac{1}{\epsilon} \frac{\partial \phi}{\partial X}\right)\right] \\
+ & \left(\frac{\partial}{\partial z}+\frac{1}{\epsilon} \frac{\partial}{\partial Z}\right)\left[K_{c}(X, Z)\left(\frac{\partial \phi}{\partial z}+\frac{1}{\epsilon} \frac{\partial \phi}{\partial Z}\right)\right]=0 .
\end{aligned}
$$

At $\mathcal{O}\left(\epsilon^{-2}\right)$ we find $\mathcal{L} \phi_{0}=0$ where $\mathcal{L}$ is a linear operator given by

$$
\mathcal{L}=\frac{\partial}{\partial X}\left(K_{c} \frac{\partial}{\partial X}\right)+\frac{\partial}{\partial Z}\left(K_{c} \frac{\partial}{\partial Z}\right) .
$$

This is solved by a function $\phi_{0}=\phi_{0}(x, z)$ that is independent of the fast scales $X$ and $Z$.

At $\mathcal{O}\left(\epsilon^{-1}\right)$ we find that

$$
\mathcal{L} \phi_{1}=-\left(\frac{\partial K_{c}}{\partial X} \frac{\partial \phi_{0}}{\partial x}+\frac{\partial K_{c}}{\partial Z} \frac{\partial \phi_{0}}{\partial z}\right) \text {. }
$$

For use in the boundary condition we note that this equation can also be expressed as

$$
\frac{\partial}{\partial X}\left[K_{c}\left(\frac{\partial \phi_{0}}{\partial x}+\frac{\partial \phi_{1}}{\partial X}\right)\right]+\frac{\partial}{\partial Z}\left[K_{c}\left(\frac{\partial \phi_{0}}{\partial z}+\frac{\partial \phi_{1}}{\partial Z}\right)\right]=0 .
$$

This is solved by a function

$$
\phi_{1}=\theta_{1}(X, Z) \frac{\partial \phi_{0}}{\partial x}+\theta_{2}(X, Z) \frac{\partial \phi_{0}}{\partial z},
$$

where the coefficients $\theta_{1}$ and $\theta_{2}$ satisfy the two cell problems

$$
\mathcal{L} \theta_{1}=-\frac{\partial K_{c}}{\partial X}, \quad \mathcal{L} \theta_{2}=-\frac{\partial K_{c}}{\partial Z} .
$$

At $\mathcal{O}(1)$ after some manipulations we find that $\phi_{0}$ satisfies the general elliptic problem

$$
\alpha \frac{\partial^{2} \phi_{0}}{\partial x^{2}}+2 \beta \frac{\partial^{2} \phi_{0}}{\partial x \partial z}+\gamma \frac{\partial^{2} \phi_{0}}{\partial z^{2}}=0,
$$

where the coefficients are given by

$$
\begin{aligned}
& \alpha=\left\langle\left\langle K_{c}\right\rangle\right\rangle-\left\langle\left\langle K_{c}\left|\nabla \theta_{1}\right|^{2}\right\rangle\right\rangle, \\
& \beta=-\left\langle\left\langle K_{c} \nabla \theta_{1} \cdot \nabla \theta_{2}\right\rangle\right\rangle,
\end{aligned}
$$

$$
\gamma=\left\langle\left\langle K_{c}\right\rangle\right\rangle-\left\langle\left\langle K_{c}\left|\nabla \theta_{2}\right|^{2}\right\rangle\right\rangle,
$$

where $\langle\langle\cdot\rangle\rangle=\int_{0}^{1} \int_{0}^{1} \cdot d X d Z$. Further details involved in this calculation can be found in Holmes. ${ }^{22}$

We now address the boundary condition (123). With the introduction of two new independent variables $X$ and $Z$, we must identify a plausible way to address the boundary condition originally applied at $z=h(x, t)$. Our approach is as follows. We allow the interface position to depend on $X$, but not on $Z$. In particular, we write $z=h(x, X, t)$. We note, however, that the terms $u$ and $w$ appearing in the boundary condition have as their arguments $u(x, z, X, Z)$ and $w(x, z, X, Z)$ to be evaluated at $z=h$. If we "preaverage" $u$ and $w$ in the $Z$ direction before inserting them into the boundary condition, we have

$$
\frac{\partial h}{\partial t}+\langle u\rangle_{Z} \frac{\partial h}{\partial x}=\langle w\rangle_{Z}, \quad \text { at } z=h(x, X, t),
$$

so that, the quantities $\langle u\rangle_{Z}$ and $\langle w\rangle_{Z}$ depend only on the variables $x, X$, and $t$. The subscript, on average, indicates with respect to what variable the average is taken. We shall indicate this subscript only where necessary. Equation (135) will then be the starting point for the homogenization techniques to be applied in the $X$ direction.

At $\mathcal{O}\left(\epsilon^{-1}\right)$ the boundary condition is satisfied by taking $h_{0}=h_{0}(x, t)$. At $\mathcal{O}(1)$ the boundary condition can be written as

$$
\frac{\partial h_{0}}{\partial t}+\left\langle u_{0}\right\rangle_{Z}\left(\frac{\partial h_{0}}{\partial x}+\frac{\partial h_{1}}{\partial X}\right)=\left\langle w_{0}\right\rangle_{Z}
$$

If we average this in $X$ we have

$$
\begin{aligned}
\frac{\partial h_{0}}{\partial t} & +\left\langle\left\langle u_{0}\right\rangle_{Z}\right\rangle_{X} \frac{\partial h_{0}}{\partial x}-\left\langle\left\langle K_{c}\left(\frac{\partial \phi_{0}}{\partial x}+\frac{\partial \phi_{1}}{\partial X}\right)\right\rangle_{Z} \frac{\partial h_{1}}{\partial X}\right\rangle_{X} \\
& =\left\langle\left\langle w_{0}\right\rangle_{Z}\right\rangle_{X},
\end{aligned}
$$

where in the third term on the left-hand side we have expressed the form of the velocity $\left\langle u_{0}\right\rangle_{Z}$ in terms of $\phi$. Note that in general the leading-order velocity components are

$$
\begin{aligned}
& u_{0}(x, z, X, Z)=-K_{c}(X, Z)\left(\frac{\partial \phi_{0}}{\partial x}+\frac{\partial \phi_{1}}{\partial X}\right), \\
& w_{0}(x, z, X, Z)=-K_{c}(X, Z)\left(\frac{\partial \phi_{0}}{\partial z}+\frac{\partial \phi_{1}}{\partial Z}\right) .
\end{aligned}
$$

We now note that the third term on the left-hand side of Eq. (137) vanishes:

$$
\begin{gathered}
\left\langle\left\langle K_{c}\left(\frac{\partial \phi_{0}}{\partial x}+\frac{\partial \phi_{1}}{\partial X}\right)\right\rangle_{Z} \frac{\partial h_{1}}{\partial X}\right\rangle_{X} \\
=-\left\langle h_{1} \frac{\partial}{\partial X}\left\langle K_{c}\left(\frac{\partial \phi_{0}}{\partial x}+\frac{\partial \phi_{1}}{\partial X}\right)\right\rangle_{Z}\right\rangle_{X}, \\
=-\left\langle h_{1}\left\langle\frac{\partial}{\partial X}\left[K_{c}\left(\frac{\partial \phi_{0}}{\partial x}+\frac{\partial \phi_{1}}{\partial X}\right)\right]\right\rangle_{Z}\right\rangle_{X},
\end{gathered}
$$




$$
\begin{aligned}
& =\left\langle h_{1}\left\langle\frac{\partial}{\partial Z}\left[K_{c}\left(\frac{\partial \phi_{0}}{\partial z}+\frac{\partial \phi_{1}}{\partial Z}\right)\right]\right\rangle_{Z}\right\rangle_{X}, \\
& =\left\langle h_{1}\left[K_{c}\left(\frac{\partial \phi_{0}}{\partial z}+\frac{\partial \phi_{1}}{\partial Z}\right)\right]_{Z=0}^{Z=1}\right\rangle_{X}=0 .
\end{aligned}
$$

The first line follows from integration by parts noting that the boundary term (evaluated at $X=0$ and $X=1$ ) vanish by periodicity in $X$, the second line by exchanging the order of differentiation with respect to $X$ and integration with respect to $Z$, the third line from Eq. (128), and the fourth line by integration in $Z$ and applying periodicity in $Z$.

The full set of averaged leading-order equations are

$$
\begin{aligned}
& \left\langle\left\langle u_{0}\right\rangle\right\rangle=-\alpha \frac{\partial \phi_{0}}{\partial x}-\beta \frac{\partial \phi_{0}}{\partial z}, \\
& \left\langle\left\langle w_{0}\right\rangle\right\rangle=-\beta \frac{\partial \phi_{0}}{\partial x}-\gamma \frac{\partial \phi_{0}}{\partial z}, \\
& 0=\frac{\partial\left\langle\left\langle u_{0}\right\rangle\right\rangle}{\partial x}+\frac{\partial\left\langle\left\langle w_{0}\right\rangle\right\rangle}{\partial z},
\end{aligned}
$$

subject to the free surface boundary condition

$$
\frac{\partial h_{0}}{\partial t}+\left\langle\left\langle u_{0}\right\rangle\right\rangle \frac{\partial h_{0}}{\partial x}=\left\langle\left\langle w_{0}\right\rangle\right\rangle, \quad \text { at } z=h_{0}(x, t),
$$

where $\alpha, \beta$, and $\gamma$ are given by Eqs. (132)-(134).

\section{F. Similarity scalings for the averaged dynamics: Two-dimensional periodicity}

To make contact to the similarity analyses presented in Sec. III we now subject Eqs. (144)-(147) to the limit $H$ $\ll R$. The scaling choices here are exactly the same as in the previous vertical and horizontal layer cases. We note that as in the case of purely vertical variation in the permeability function one must keep in mind that the horizontal and vertical length scales are different when specifying $K_{c}$. The presence of derivatives of $\phi$ with respect to both $x$ and $z$ leads to an extra step required in the identification of the averaged velocities. We expand $\phi_{0}=\phi_{0}^{0}+\delta \phi_{0}^{1}+\cdots$, where $\delta=H / R$, and again find that $\phi_{0}^{0}$ is independent of $z$. It follows from the boundary condition $\phi=h$ on $z=h$ that $\phi_{0}^{0}$ $=h_{0}(x, t)$. At the next order we find from Eqs. (144) and (145) that

$$
\begin{aligned}
& \left\langle\left\langle u_{0}\right\rangle\right\rangle=-\alpha \frac{\partial \phi_{0}^{0}}{\partial x}-\beta \frac{\partial \phi_{0}^{1}}{\partial z}, \\
& 0=-\beta \frac{\partial \phi_{0}^{0}}{\partial x}-\gamma \frac{\partial \phi_{0}^{1}}{\partial z} .
\end{aligned}
$$

We can solve these two equations to find

$$
\left\langle\left\langle u_{0}^{0}\right\rangle\right\rangle=-\left(\alpha-\frac{\beta^{2}}{\gamma}\right) \frac{\partial \phi_{0}^{0}}{\partial x} .
$$

It follows from the continuity equation that the vertical velocity is given by

$$
\left\langle\left\langle w_{0}^{0}\right\rangle\right\rangle=+\left(\alpha-\frac{\beta^{2}}{\gamma}\right) \frac{\partial^{2} \phi_{0}^{0}}{\partial x^{2}} z .
$$

Inserting these results into the free surface boundary condition and noting that $\phi_{0}^{0}=h_{0}$ gives

$$
\frac{\partial h_{0}}{\partial t}=\frac{\left(\alpha \gamma-\beta^{2}\right)}{\gamma} \frac{\partial}{\partial x}\left[h_{0} \frac{\partial h_{0}}{\partial x}\right] .
$$

A comparison of this with full numerical simulations with $K_{c}=K_{c}(x, z)$ requires a numerical calculation of the coefficient $\left(\alpha \gamma-\beta^{2}\right) / \gamma$ via the cell problems (130). We leave this for future work where the validity of the formal preaveraging applied to the moving interface can be assessed. This effective permeability reduces to those previously identified for vertical and horizontal layers.

\section{A THIN ASPECT RATIO, SCALAR PDE FOR SLUMPING GRAVITY CURRENTS}

We remark that the homogenization of a moving interface is a somewhat nebulous concept, and the homogenization of such nonlinear problems certainly can be supplemented by rigorous mathematical analysis. To give a more concrete picture for what is going on, in this section, we first derive a decoupled, variable coefficient partial differential equation governing the slumping interface under the asymptotic assumption of a thin, wide gravity current, with a vertically layered background porous medium. We then, in turn, compute the leading-order homogenization, which is found to agree with the homogenized dynamics of Sec. IV B. Finally, we conclude this section with a presentation of the explicit correction to the homogenized dynamics.

In the limit $H / R \rightarrow 0$ the original governing equations are given by

$$
\begin{aligned}
& u=-K_{c}(X) \frac{\partial \phi}{\partial x}, \\
& 0=-K_{c}(X) \frac{\partial \phi}{\partial z}, \\
& 0=\frac{\partial u}{\partial x}+\frac{\partial w}{\partial z}, \\
& w(h)=\frac{\partial h}{\partial t}+u(h) \frac{\partial h}{\partial x} .
\end{aligned}
$$

Upon noting that the second of these equations leads to the result that $\phi=\phi(x, X, t)$ we find that $\phi=h(x, X, t)$ from boundary condition (11).

We are interested in examining this problem using the multiscale method and averaging over the fast variations in $X$ to get a leading-order effective problem but then also obtaining corrections to this leading-order behavior that show details of the fast variations. This method has been developed and applied to a single PDE. Here we aim to understand how such a method can be applied when there is a free boundary in the problem. The above thin limit offers a convenient setting in which to examine this issue as the system in this case can be reduced to a single PDE for the interface position $h(x, X, t)$. This equation is given by 


$$
\frac{\partial h}{\partial t}=\frac{\partial}{\partial x}\left[K_{c}(X) h \frac{\partial h}{\partial x}\right]
$$

and is subject to the conditions $\partial h / \partial x=0$ at $x=0$ and $h$ $=0$ at $x=R$. As noted earlier, the evolution of the contact line position $R$ is determined by examining Eq. (157) and the condition $h=0$ at the contact line. That is, computing the dynamics of $h$ according to Eq. (157) will effectively determine the dynamics of $R$ by applying $h=0$ at $x=R$. Our focus, then, will be on the dynamics of $h$.

We expand Eq. (157) using multiscale methods and obtain at $\mathcal{O}\left(\epsilon^{-2}\right)$ that

$$
0=\frac{\partial}{\partial X}\left[K_{c}(X) h_{0} \frac{\partial h_{0}}{\partial X}\right],
$$

which indicates that $h_{0}=h_{0}(x, t)$. At $\mathcal{O}\left(\epsilon^{-1}\right)$ we find that

$$
\begin{aligned}
0= & \frac{\partial}{\partial X}\left[K_{c}(X)\left(h_{0} \frac{\partial h_{1}}{\partial X}+h_{1} \frac{\partial h_{0}}{\partial X}\right)\right]+\frac{\partial}{\partial x}\left[K_{c}(X) h_{0} \frac{\partial h_{0}}{\partial X}\right] \\
& +\frac{\partial}{\partial X}\left[K_{c}(X) h_{0} \frac{\partial h_{0}}{\partial x}\right] .
\end{aligned}
$$

It follows from this equation that

$$
K_{c}(X) h_{0}\left[\frac{\partial h_{1}}{\partial X}+\frac{\partial h_{0}}{\partial x}\right]=a(x, t),
$$

where, upon averaging and using $h_{1}(X=0)=h_{1}(X=1)$, we find that the function $a(x, t)$ is given by

$$
a(x, t)=\left\langle K_{c}^{-1}\right\rangle^{-1} h_{0} \frac{\partial h_{0}}{\partial x} .
$$

It also follows that

$$
h_{1}(x, X, t)=\frac{\partial h_{0}}{\partial x} \int_{0}^{X}\left[\frac{K_{c}^{-1}}{\left\langle K_{c}^{-1}\right\rangle}-1\right] d X+b(x, t),
$$

where $b(x, t)$ is a function to be determined.

At $\mathcal{O}(1)$,

$$
\begin{aligned}
\frac{\partial h_{0}}{\partial t}= & \frac{\partial}{\partial X}\left[K_{c}(X)\left(h_{0} \frac{\partial h_{2}}{\partial X}+h_{1} \frac{\partial h_{1}}{\partial X}+h_{2} \frac{\partial h_{0}}{\partial X}\right)\right] \\
& +\frac{\partial}{\partial x}\left[K_{c}(X)\left(h_{0} \frac{\partial h_{1}}{\partial X}+h_{1} \frac{\partial h_{0}}{\partial X}\right)\right] \\
& +\frac{\partial}{\partial X}\left[K_{c}(X)\left(h_{0} \frac{\partial h_{1}}{\partial x}+h_{1} \frac{\partial h_{0}}{\partial x}\right)\right] \\
& +\frac{\partial}{\partial x}\left[K_{c}(X) h_{0} \frac{\partial h_{0}}{\partial x}\right] .
\end{aligned}
$$

Upon averaging this equation we find that the leading-order interface shape $h_{0}(x, t)$ satisfies the equation

$$
\frac{\partial h_{0}}{\partial t}=\left\langle K_{c}^{-1}\right\rangle^{-1} \frac{\partial}{\partial x}\left[h_{0} \frac{\partial h_{0}}{\partial x}\right] \text {. }
$$

This equation can be compared to Eq. (40), where we note that here $\left\langle K_{c}^{-1}\right\rangle^{-1}$ replaces the constant $K_{c}$ in (40). We can also integrate Eq. (163) to obtain

$$
h_{0}\left(\frac{\partial h_{2}}{\partial X}+\frac{\partial h_{1}}{\partial x}\right)+h_{1}\left(\frac{\partial h_{1}}{\partial X}+\frac{\partial h_{0}}{\partial x}\right)=\frac{\tilde{c}(x, t)}{K_{c}(X)},
$$

where $\tilde{c}(x, t)$ is a function to be determined. We can determine $\tilde{c}(x, t)$ by averaging Eq. (165) to obtain

$$
h_{0}\left\langle\frac{\partial h_{1}}{\partial x}\right\rangle=-\left\langle h_{1}\right\rangle \frac{\partial h_{0}}{\partial x}+\tilde{c}\left\langle K_{c}^{-1}\right\rangle,
$$

while the solution for $h_{1}$ in Eq. (162) gives

$$
\left\langle\frac{\partial h_{1}}{\partial x}\right\rangle=\frac{\partial^{2} h_{0}}{\partial x^{2}}\left\langle\int_{0}^{X}\left(\frac{K^{-1}}{\left\langle K^{-1}\right\rangle}-1\right) d X\right\rangle+b_{x} .
$$

These two results together give an expression for $\tilde{c}$,

$$
\widetilde{c}(x, t)\left\langle K_{c}^{-1}\right\rangle=\frac{\partial\left(b h_{0}\right)}{\partial x}+\langle X\rangle \frac{\partial}{\partial x}\left[h_{0} \frac{\partial h_{0}}{\partial x}\right]-\frac{\partial h_{0}}{\partial t}\left\langle\frac{X}{K_{c}}\right\rangle .
$$

At $\mathcal{O}(\epsilon)$

$$
\begin{aligned}
\frac{\partial h_{1}}{\partial t}= & \frac{\partial}{\partial X}\left[K_{c}(X)\left(h_{0} \frac{\partial h_{3}}{\partial X}+h_{1} \frac{\partial h_{2}}{\partial X}+h_{2} \frac{\partial h_{1}}{\partial X}+h_{3} \frac{\partial h_{0}}{\partial X}\right)\right] \\
& +\frac{\partial}{\partial x}\left[K_{c}(X)\left(h_{0} \frac{\partial h_{2}}{\partial X}+h_{1} \frac{\partial h_{1}}{\partial X}+h_{2} \frac{\partial h_{0}}{\partial X}\right)\right] \\
& +\frac{\partial}{\partial X}\left[K_{c}(X)\left(h_{0} \frac{\partial h_{2}}{\partial x}+h_{1} \frac{\partial h_{1}}{\partial x}+h_{2} \frac{\partial h_{0}}{\partial x}\right)\right] \\
& +\frac{\partial}{\partial x}\left[K_{c}(X)\left(h_{0} \frac{\partial h_{1}}{\partial x}+h_{1} \frac{\partial h_{0}}{\partial x}\right)\right] .
\end{aligned}
$$

Upon averaging this equation, using Eq. (165), and periodicity of the solutions, we find that

$$
\frac{\partial}{\partial t}\left\langle h_{1}\right\rangle=\frac{\partial \widetilde{c}}{\partial x}
$$

This equation can be written in terms of the unknown function $b(x, t)$ as

$$
\frac{\partial b}{\partial t}=\left\langle K_{c}^{-1}\right\rangle^{-1} \frac{\partial^{2}\left(b h_{0}\right)}{\partial x^{2}} .
$$

We note that this equation for $b$ is the equation for $h_{0}$ linearized about $h_{0}$, so that we can write

$$
h(x, X, t)=\tilde{h}_{0}(x, t)+\epsilon h_{1}^{(p)}(x, X, t),
$$

where $\tilde{h}_{0}(x, t)=h_{0}(x, t)+\epsilon b(x, t)$ satisfies

$$
\frac{\partial \tilde{h}_{0}}{\partial t}=\left\langle K_{c}^{-1}\right\rangle^{-1} \frac{\partial}{\partial x}\left[\tilde{h}_{0} \frac{\partial \tilde{h}_{0}}{\partial x}\right]
$$

and $h_{1}^{(p)}$ is given from Eq. (162) by

$$
h_{1}^{(p)}=\frac{\partial h_{0}}{\partial x} \int_{0}^{X}\left[\frac{K_{c}^{-1}}{\left\langle K_{c}^{-1}\right\rangle}-1\right] d X .
$$

We note that the correction given in Eq. (174) depends upon the homogenized solution, $h_{0}(x, t)$, as well as the explicit structure of the underlying porous media. A compari- 


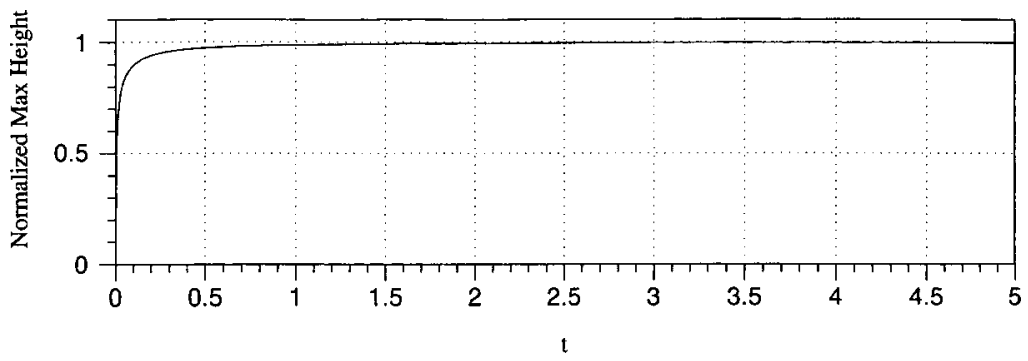

FIG. 1. Approach (A): Maximum of $h(x, t)$ versus time, normalized by the similarity solution with $K_{0}$ $=0.5$ and using the effective permeability $K_{c}^{*}$ $=0.866025$ in the similarity solution.

son with the general aspect ratio correction for $h$ will be explored in a subsequent work devoted to the full twodimensional analysis and simulations.

\section{COMPUTATIONAL SIMULATIONS}

\section{A. Numerical approximation approaches}

In the previous three sections, we explored the theoretical averaging of the evolving gravity current under the assumption of rapidly varying, vertically and/or horizontally, layered porous media. In the case of vertically layered media, we derived, in addition, explicit corrections valid for thin aspect ratios. Here we present computational simulations, which demonstrate three points made in this article, applied to the special case of the thin aspect ratio variable coefficient dynamics. First, we document that from a general, non-self-similar initial condition, the self-similar scaling emerges in finite time for the case of a variable coefficient permeability through normalization by the similarity solution employing an effective, homogenized permeability. Second, we further document that the leading-order averaging theory emerges at finite time, and finite $\epsilon$ (wavelength) here through numerical comparison of the front profiles between the full solution with variable coefficient permeability and that of the homogenized dynamics. Last, we explicitly show that the theoretical corrections to the leading-order homogenized dynamics are accurate as regards the simulations at both finite time, and fixed, finite $\epsilon$ in the interior, away from the contact line region.

We consider the numerical simulation of the following partial differential equation:

$$
\frac{\partial h}{\partial t}=\frac{\partial}{\partial x}\left[\frac{\partial h}{\partial x} \int_{0}^{h} K_{c}(x / \epsilon, z / \epsilon) d z\right],
$$

which allows a general hydraulic conductivity function $K_{c}(x / \epsilon, z / \epsilon)$. This equation simplifies depending on the specific form for $K_{c}$ and is not an integrodifferential equation. The variable $z$ here is scaled with respect to the thin geometry and so represents a special case of the two-dimensional periodicity described earlier. We examine two special cases of variability in one dimension by exploring the solution of this equation for cases with vertical layers and with horizontal layers. We shall implement two distinct numerical approaches: one is an explicit method that avoids direct application of contact line boundary conditions and the second is an implicit method that incorporates the contact line boundary conditions. Below we outline these methods and results of the calculations.
In approach (A) we solve Eq. (175) and attempt to simplify the handling of the contact line condition. Rather than imposing directly the contact line conditions we take an initial profile that is a tight Gaussian:

$$
h(x, 0)=\beta_{0} e^{-(x-L / 2)^{2} / \alpha_{0}},
$$

applied over the entire computational domain of length $L$. The contact line conditions are then effectively replaced by a region of small but nonzero values of $h$ extending to the end of the computational domain. Furthermore, we do not enforce symmetry. Unless otherwise noted, we take $L=20$, the amplitude $\beta_{0}=10$, and the width, $\alpha_{0}=0.75$. The permeability function is given by $K_{c}(x / \epsilon)=1+K_{0} \sin 2 \pi x / \epsilon$ corresponding to vertically oriented layers. Unless otherwise noted, we utilize the value of $\epsilon=0.5$, which corresponds to roughly 40 oscillations over the spatial domain. Further, for the initial data considered, the initial data spans roughly eight periods of the permeability field. Since this is a onedimensional problem we use an explicit time-stepping scheme with an extremely small time step to compute the solution. Unless otherwise noted, all simulations for approach (A) are run until time $t=5.0$ using $1.2 \times 10^{7}$ time steps, and a uniform spatial mesh with 2400 spatial nodes. By this output time, the gravity current is very well defined and has not reached the end of the computational domain.

In approach (B) we impose directly the boundary conditions (6) and (7). The contact line condition,

$$
\frac{d R}{d t}=u(R, t)=-\left.K_{c}(R / \epsilon) \frac{\partial h}{\partial x}\right|_{x=R},
$$

is also directly enforced. Consistent with the imposed symmetry, we choose the even function $K_{c}(x / \epsilon)=1$ $+K_{0} \cos (2 \pi x / \epsilon)$ for the permeability, where $K_{0}$ and $\epsilon$ are given constants. The initial conditions are $h(x, 0)=A_{H}(1$ $-x^{2}$ ) and $R(0)=1$, where $A_{H}$ is a given constant. We solve this system numerically using a finite difference approach with second-order accurate central differencing in space and a backward Euler implicit time stepping routine. The details are given in Appendix B.

\section{B. Vertically layered case}

In Fig. 1, using approach (A), we document the rapid convergence to the similarity scaling given in (44) by taking the maximum of the computed field $h(x, t)$ and normalizing by the similarity scaling given in Eq. (44). In this simulation, the variable coefficient permeability has amplitude $K_{0}=\frac{1}{2}$. The effective permeability, in this geometry given by the 


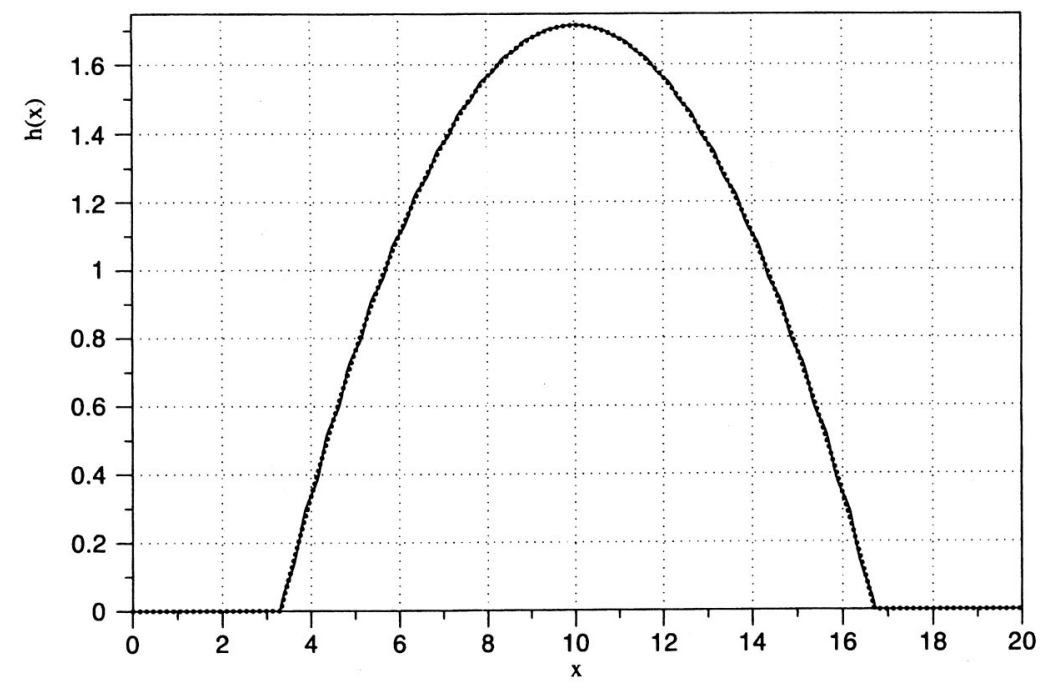

FIG. 2. Approach (A): A comparison between the spatial profiles at time, $t=5$, between the simulated complete solution, and simulated homogenized solution, with $K_{0}=0.5$, and $K_{c}^{*}=0.866025$

harmonic mean, is $K_{c}^{*}=0.866025$. Observe that the convergence to unity is very rapid, indicating that the similarity scaling is reached quickly, and that the system, at least as regards the maximum, is homogenizing quickly as well.

In Fig. 2, we show a comparison between the spatial profiles of the height field at the final time of $t=5$. Observe, the unaveraged solution oscillates rapidly (with a wavelength set by the intrinsic period of the underlying permeability) about the simulated effective dynamics. Clearly, the homogenized dynamics are doing an excellent job in capturing the dynamics.

In Figs. 3 and 4, we document the remarkable success of the correction to the leading-order homogenized dynamics in capturing the true behavior given by the full unaveraged simulation. Define $e(x, t)=h(x, t)-h_{0}(x, t)$, where $h_{0}(x, t)$ is the homogenized solution. Figure 3 shows the spatial profile of the field $e(x, t)$ at time $t=5$. Observe there is a periodscale oscillation with amplitude growing roughly linearly from the center, with an abrupt drop off at the edge of the current. The linear envelope can be understood by noting that $h_{1} \sim \partial h_{0} / \partial x$ [see Eq. (174)] and that $h_{0}$ is approximately parabolic.

To make a direct comparison with the theoretical prediction of Sec. IV B, we numerically process the correction given in Eq. (174), since it involves the nonlinear, homogenized dynamics. However, since we have already computed the homogenized solution (at a greatly diminished cost as compared with that necessary to resolve the rapid oscilla- tions in the unaveraged dynamics), this task is automatic. Define $E(x, t)=e(x, t)-h_{1}(x, t)$. The field $E(x, t)$ denotes the difference between the theoretical prediction and the full simulation of the unaveraged dynamics. In Fig. 4, we document the success of the homogenization approach in this simple case of a scalar nonlinear variable coefficient PDE by showing the field $E(x, t)$ at time $t=5$. Observe that essentially across the entire spatial domain, the theoretical prediction is extremely good, with the error possibly accumulating at the edge of the gravity current. This error is qualitatively similar to that computed using approach (B), see below, where the contact line is directly incorporated in the calculation.

Calculations using approach (B) are shown in Figs. 5-8. In Fig. 5(a) we show a comparison of the full numerical solution $h(x, t)$ and two approximate solutions of the form $h_{0}+\epsilon h_{1}$. One approximate solution calculates $h_{0}$ numerically using Eq. (175) with the variable $K_{c}$ replaced by the constant effective value $\left\langle K_{c}^{-1}\right\rangle^{-1}$. The derivative of $h_{0}$ appearing in the analytical formula for $h_{1}$ [see Eq. (174)] is calculated using this $h_{0}$ solution. This is the approximation based on the leading-order numerical solution. The other approximate solution uses the similarity solution for $h_{0}$, again with the value of $K_{c}$ taken to be the constant effective value. The derivative $\partial h_{0} / \partial x$ in the formula for $h_{1}$ is obtained from the similarity solution. This is the approximation based on the similarity solution. As can be seen from this figure, the

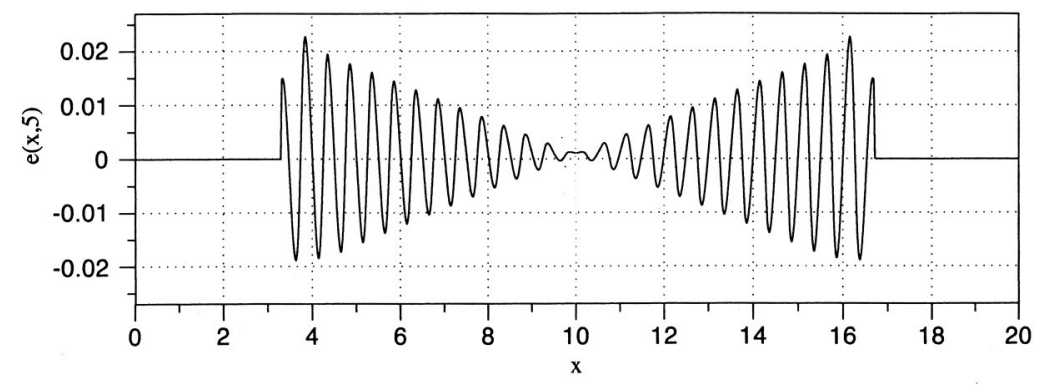

FIG. 3. Approach (A): Difference between the full unaveraged simulation and the homogenized simulation at $t=5$. 


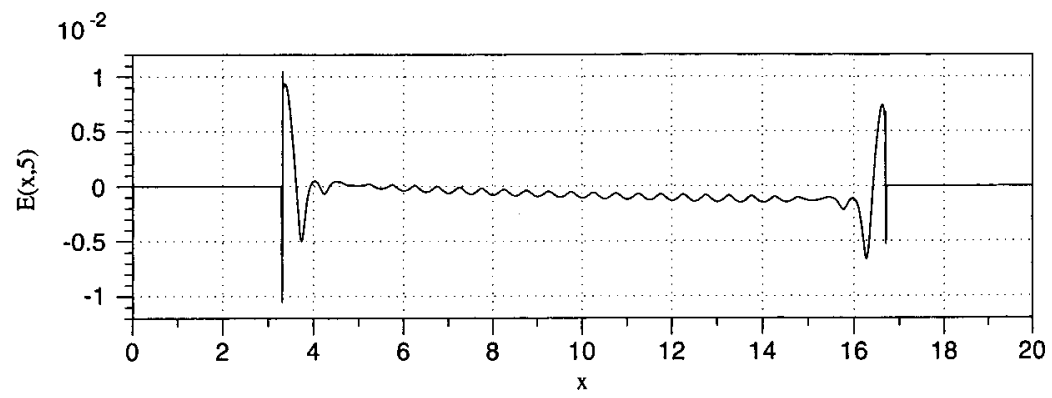

FIG. 4. Approach (A): The simulated error field, $E(x, t)$, at $t=5$.

approximate solutions appear to agree well with the full numerical solution.

In Fig. 5(b) we show the difference between the full numerical solution and the leading-order $h_{0}, e(x, t)$ $=h(x, t)-h_{0}(x, t)$, based on the leading-order numerical solution (solid line) and the similarity solution (dashed line). These curves should approximate the known correction formula, shown in Fig. 5(c). Note that Fig. 5(c) shows the analytical correction calculated with both the leading-order numerical solution and the similarity solution, indicating very good agreement between the two.

The difference between the curves in Fig. 5(b) and those in Fig. 5(c) is $E(x, t)=h(x, t)-h_{0}(x, t)-\epsilon h_{1}(x, t)$ and is shown in Fig. 5(d). Again, the solid curve shows the difference based on the leading-order numerical solution and the dashed line shows that based on the similarity solution. This error indicates very good agreement between numerical and approximate solutions in the interior region. The error is better in the interior, in general, in all simulations using the explicit approach (A) and the implicit approach (B). This trend remains with increased spatial and temporal resolution.

In Fig. 6 we quantify the dependence of the errors $e(x, t)=h(x, t)-h_{0}(x, t) \quad$ and $\quad E(x, t)=h(x, t)-h_{0}(x, t)$ $-\epsilon h_{1}(x, t)$ on $\epsilon$. Since there is a computational trade-off between the end point in time $t$ for the calculation and the spatial resolution used, we have chosen to make this error assessment at a relatively early time $t=0.1$ in order to make feasible computations with smaller values of $\epsilon$ and more spatial resolution. Consequently, we use the leading-order numerically computed solution rather than the similarity solu- (a)
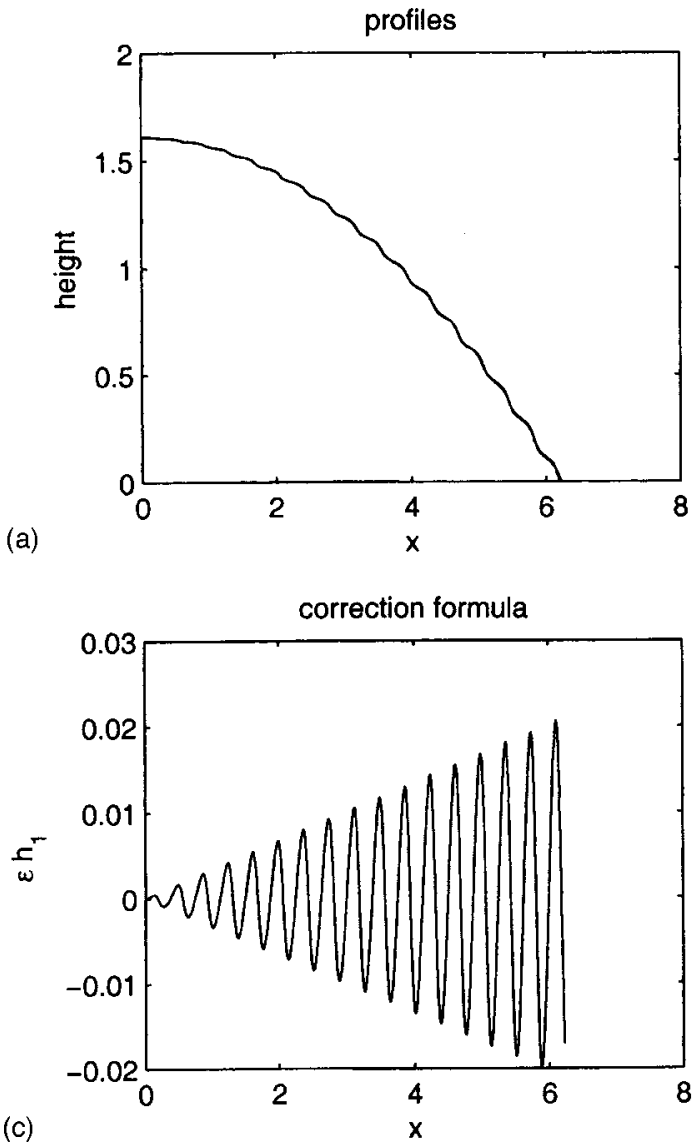
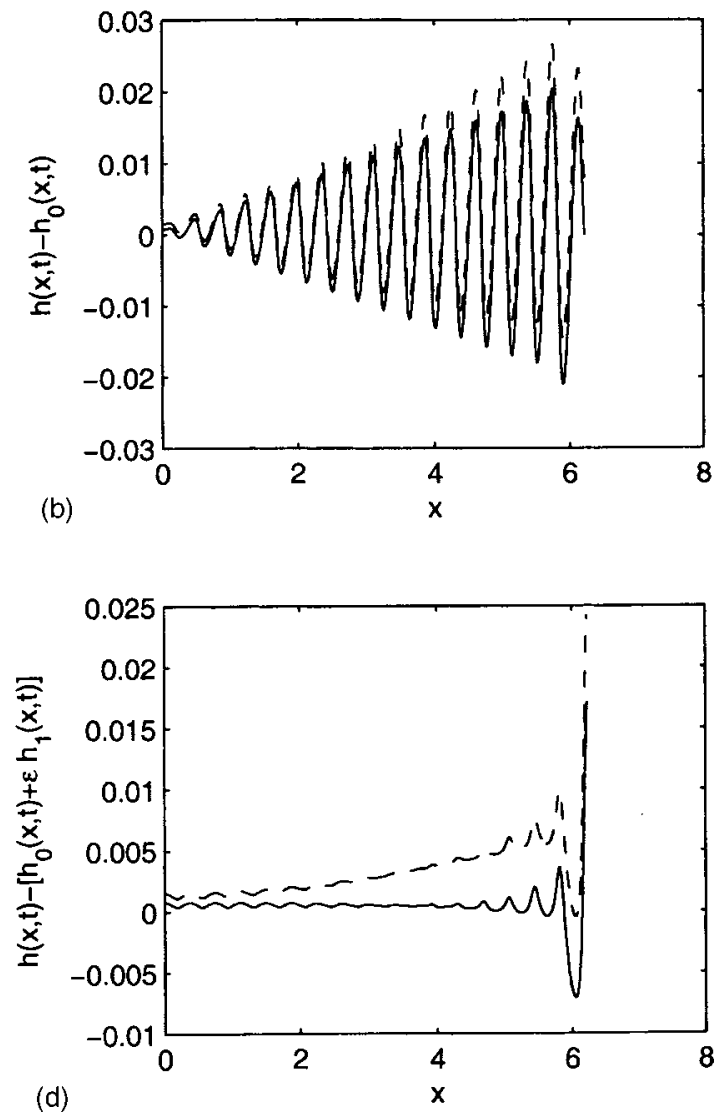

FIG. 5. Approach (B): This figure shows (a) the solution profiles, (b) the difference $e(x, t)=h(x, t)-h_{0}(x, t)$, (c) the correction formula $\epsilon h_{1}(x, t)$, and (d) the difference $E(x, t)=h(x, t)-h_{0}(x, t)-\epsilon h_{1}(x, t)$. See the text for an explanation of the different curves shown. The numerical solutions shown here have been computed using $n t=10000, n x=1000, K_{0}=0.6, \epsilon=0.375$, and $A_{H}=10$. Note that $K_{c}^{*}=\left\langle K_{c}^{-1}\right\rangle^{-1}=0.8$ in this case. 


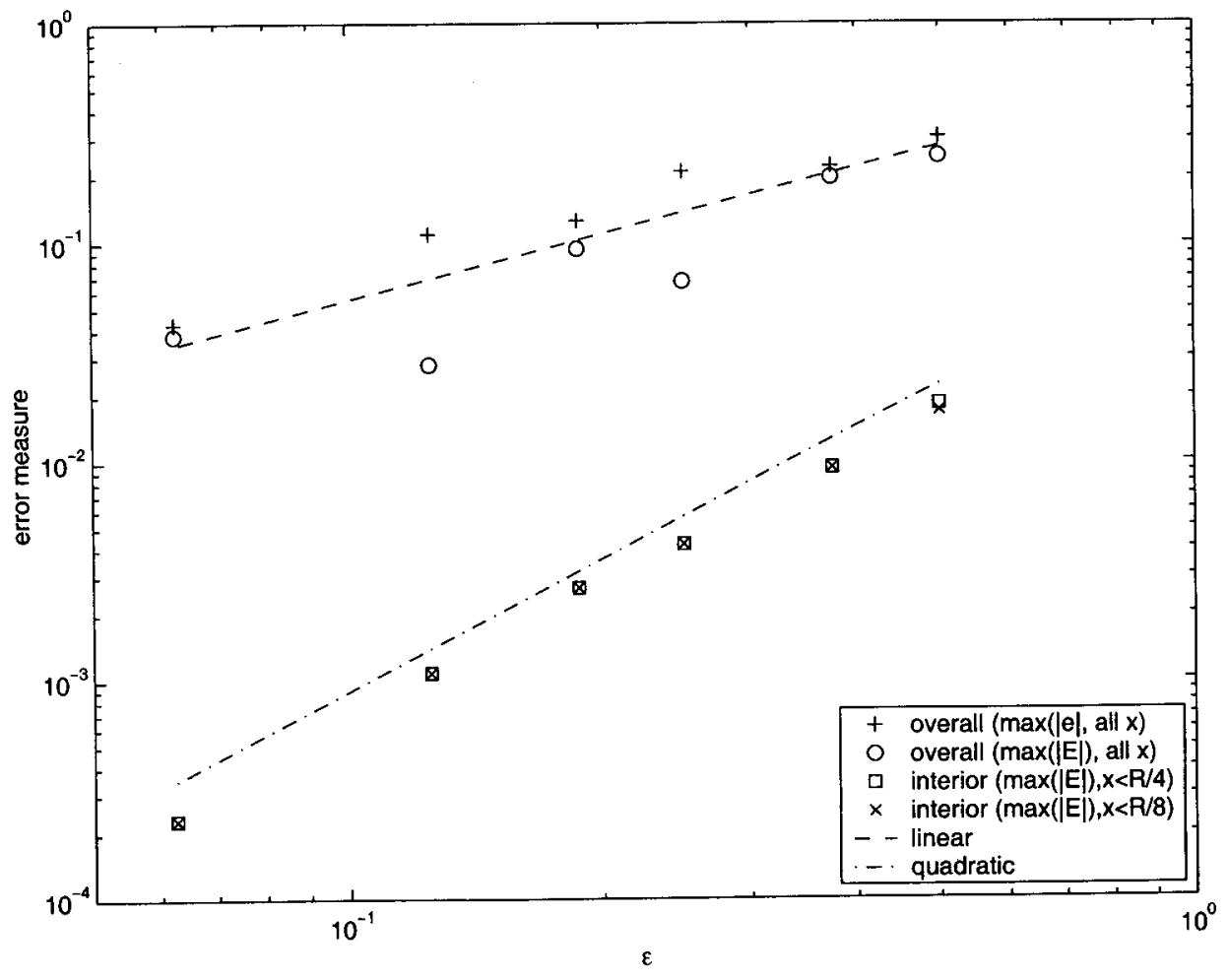

FIG. 6. Approach (B): This figure shows different measures of error as reduced from error fields similar to those shown in Figs. 5(b), 5(d). This set of error data has been calculated at an early time $t=0.1$. The overall error fields $e(x, t)$ and $E(x, t)$ both decay linearly with $\epsilon$. However, the interior error $E(x, t)$ measured on the intervals $x<R / 4$ and $x<R / 8$ suggest quadratic decay in $\epsilon$ in the interior. The quantity $\max (|E(x, t)|)$ measured over all $x$ really measures the error near the contact line as that is where the maximum error occurs and indicates nonuniform convergence of the expansion $h_{0}$ $+\epsilon h_{1}$. The numerical solutions shown here have been computed using $n t$ $=500, n x=2000, K_{0}=0.6$, and $A_{H}$ $=10$ with different values of $\epsilon$, as indicated.

tion in the comparison. We show several measures of the error. In this figure, the two drawn lines indicate linear and quadratic dependence on $\epsilon$. We first show the maximum of $|e(x, t)|$ over the spatial domain. This error decreases approximately linearly in $\epsilon$, indicating that the leading-order solution is uniformly valid over the whole domain. The maximum of $|E(x, t)|$ over the whole domain indicates a trend that is linear in $\epsilon$ also. However, we note that this is really measuring the error at the contact line, which we have already noted is larger than in the interior. Two other measures of the error $|E(x, t)|$ are shown for two interior regions $[(x<R / 4)$ and $(x<R / 8)]$, showing that this error is approximately quadratic in $\epsilon$, or at least $o(\epsilon)$. The observed nonuniformity of the error $E(x, t)=h-h_{0}-\epsilon h_{1}$ indicates that the correction formula $h_{1}$ is not uniformly valid. It is clear that the error is spatially dependent and is consistently larger near
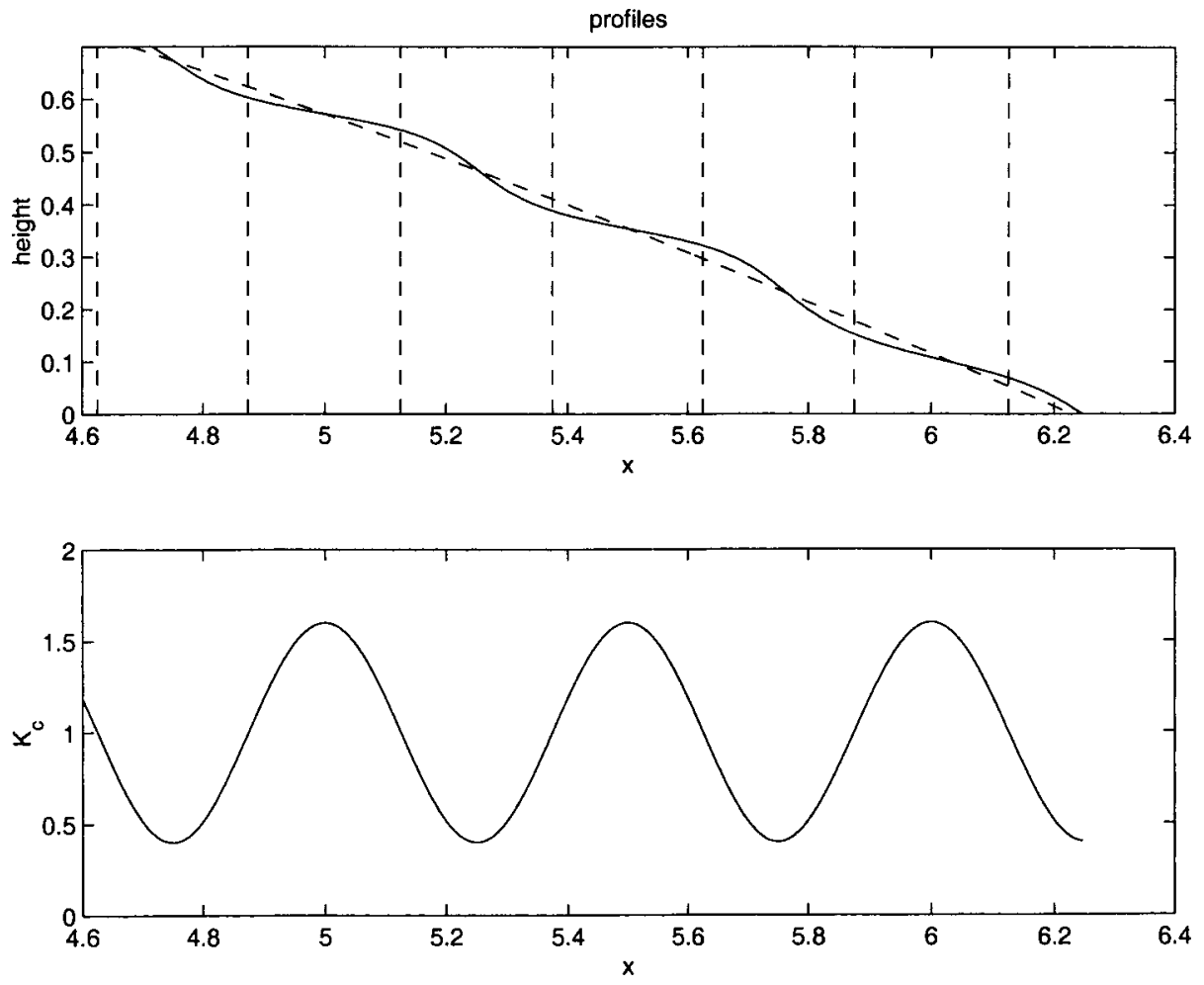

FIG. 7. Approach (B): The full solution profile (solid curve) and leadingorder solution profile (dashed curve) plotted against the background permeability in the vertical layer case. The vertical lines in the upper plot correspond to where the permeability, as shown in the lower plot, takes on its mean value. In regions of higher than mean permeability, the interface profile has a smaller slope than it does in regions of lower than mean permeability. Note that the bulges in the interface profile are localized around regions where the permeability is decreasing most rapidly. The full numerical solution in this figure has $n t$ $=10000, n x=1000, K_{0}=0.6, \epsilon=0.5$, and $A_{H}=10$. Note that $K_{c}^{*}=\left\langle K_{c}^{-1}\right\rangle^{-1}$ $=0.8$ in this case. 

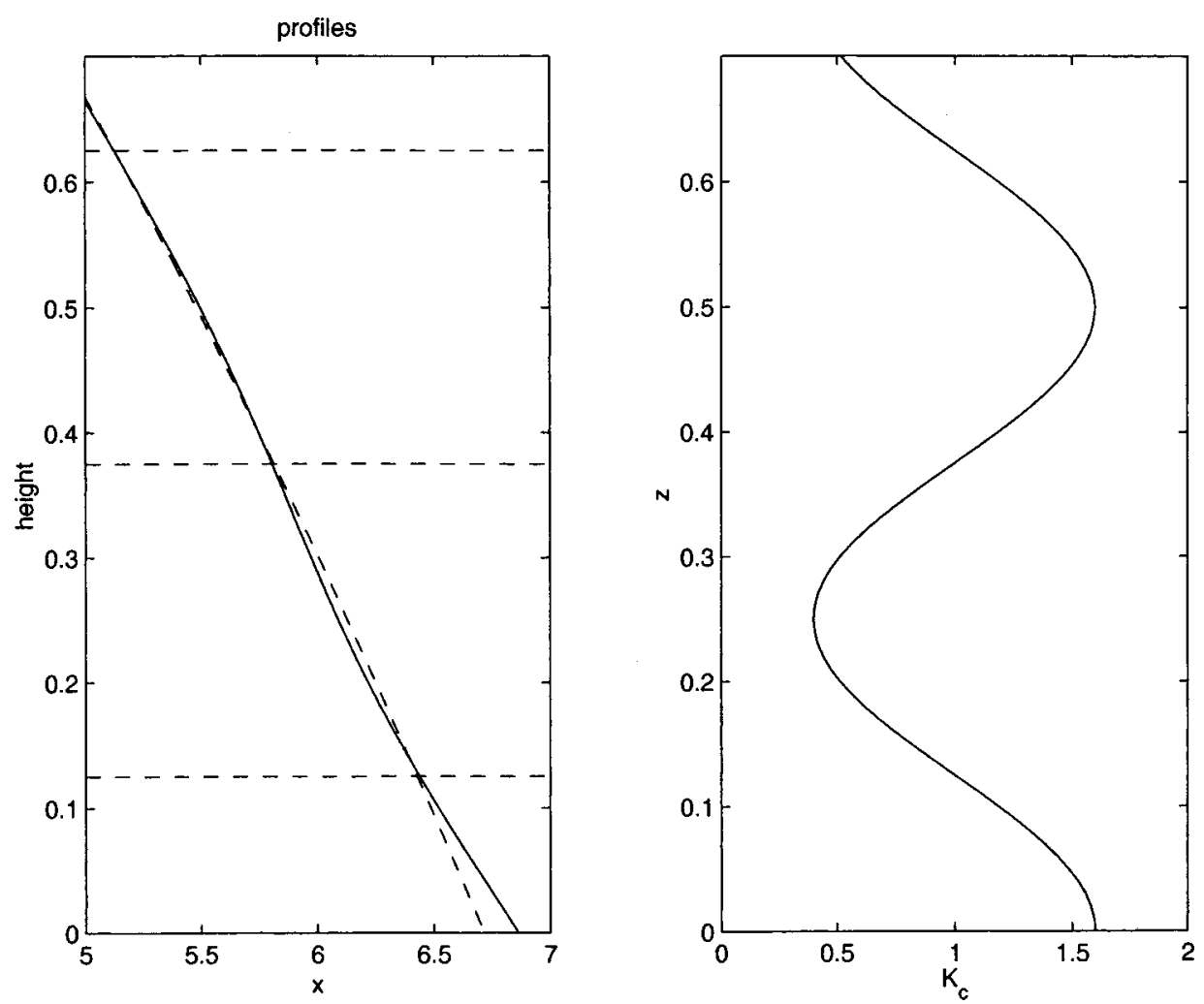

FIG. 8. Approach (B): The full solution profile (solid curve) and leadingorder solution profile (dashed curve) plotted against the background permeability in the horizontal layer case. The horizontal lines in the left plot correspond to where the permeability, as shown in the right plot, takes on its mean value 1 . This figure has $n t$ $=5000, n x=500, K_{0}=0.6, \epsilon=0.5$, and $A_{H}=10$. Note that $K_{c}^{*}=\left\langle K_{c}\right\rangle$ $=1.0$ in this case.

the contact line. The clear quadratic scaling of the error in the interior gives strong evidence that the correction formulas both are asymptotically correct, and achieve in capturing the spatial imprint imparted to the evolving interface by the medium. At the contact line, however, the corrections may not be asymptotically valid. Ultimately determining if the corrections are asymptotic at the contact line will require further simulations, and potentially different local expansion techniques. It is noteworthy that the corrections are performing correct in the interior for the interface, which is a boundary in its own right.

Figure 7 shows a close-up of the profiles with $\epsilon=0.5$ with all other parameters as shown in Fig. 5. The upper plot shows the numerically calculated solution with variable permeability (solid curve) and the numerically calculated solution with an effective permeability of $\left\langle K_{c}^{-1}\right\rangle^{-1}=0.8$ (dashed curve). Again, it is clear that the calculation with effective permeability agrees well with the bulk motion of the gravity current in the variable permeability case. The background permeability is shown in the lower figure (the vertical dashed lines in the upper plot separate regions of high and low permeabilities). We note that the bulges in the fluid profile appear to be approximately $90^{\circ}$ out of phase with the maxima in the permeability function. This shape is consistent with the analytical formula derived for the correction $h_{1}$ given by Eq. (174) and as shown in Fig. 5. The flatness of the profile in the region of high permeability indicates the fluid's greater mobility in that region.

\section{Horizontally layered case}

To test the leading-order predictions for horizontal layers, we numerically simulate a case with $K_{c}=K_{c}(z / \epsilon)$ in the slender aspect ratio limit using approach $(\mathbf{B})$. We solve Eq. (175) with $K_{c}(z / \epsilon)=1+K_{0} \cos (2 \pi z / \epsilon)$, where $z$ here is interpreted as scaled with respect to the thin geometry. Note that in this case the predictions from Sec. IV D suggest that the leading-order behavior can be obtained by replacing variable $K_{c}$ with $\left\langle K_{c}\right\rangle$. For the above choice of $K_{c}(z / \epsilon)$ we find that $\left\langle K_{c}\right\rangle=1$. Figure 8 shows on the left the numerically calculated solution with $K_{0}=0.6$ and $\epsilon=0.5$ (solid curve) and the numerically calculated solution with a constant effective permeability $\left\langle K_{c}\right\rangle=1$ (dashed curve). This leadingorder solution is clearly capturing the bulk motion of the fluid. Indicated on the right of Fig. 8 is the permeability variation used in the full simulation. We can see that in regions of lower than mean permeability, the interface lags behind the homogenized solution and in regions of higher than mean permeability the interface precedes the homogenized profile. Bulges of the interface are in phase with the permeability variations.

We point out here that based on the leading-order similarity solutions for the vertical and horizontal layer cases we can compare the spreading rates of these two situations using Eq. (54). If we represent the radius for vertically oriented layers by $R_{V}$ and that for horizontally oriented layers by $R_{H}$, we find that

$$
\frac{R_{V}}{R_{H}}=\frac{\left\langle K_{c}^{-1}\right\rangle^{-1}}{\left\langle K_{c}\right\rangle}
$$

This ratio is less than one, which implies that gravity currents flowing in horizontally layered media spread faster than the same gravity current flowing in a vertically layered media. 


\section{CONCLUSIONS}

In this article, we have explored the behavior of a slumping gravity current in the presence of a variable permeability porous medium. We have identified the similarity scalings and solutions for a constant permeability medium, and intertwined these tools with the variable permeability case through the method of homogenized averaging, specifically in cases with either vertically or horizontally layered permeabilities. For the case of two-dimensional permeability, using a more formal approach, we derive effective medium dynamics, and show how the slender limit similarity dynamics are obtained up to a coefficient, which must be obtained in general through the numerical solution of an auxiliary cell problem. Under the assumption that the gravity current is thin, we identified that the height itself completely decouples yielding a scalar, variable coefficient nonlinear PDE governing the moving interface. We analyzed this case in detail, both at the level of leading-order homogenization and with corrections for the case of vertical layers, and further documented the success of this averaging approach through computational simulations in this simplified geometry.

Many issues require further study. Clearly, general porous media are not layered as such, but will have full topology, and the study of the moving interface problem, in general, will require at least the homogenization of a nonlayered background. It is expected that the connection to the similarity scalings through homogenized averaging will be a general principle; though the calculation of the effective permeabilities themselves is a nonexplicit task in the absence of a layered structure. Moreover, identifying how the structure of the medium manifests itself upon the slumping gravity current surely will require the complete simulation of the fully coupled and multidimensional system. The assumption of periodicity should be ultimately relaxed as well if an efficient pumping strategy is to be ultimately designed to handle general porous media, and supplemented with a random permeability tensor. Homogenization methods may well work in these more complicated environments; however, a study of the averaging of the underlying elliptic operators is necessary. The averaging method for general topology for the complete system (outside of the lubrication approximation) is more formal than the averaging presented for layered systems. Future work will compare this methodology with complete simulations of the full porous media system.

The study presented here provides a first pass at assessing the relevant time scales for maintaining an elevated density profile in a porous media, and further documents the success of the leading-order plus corrected homogenization theory applied to this nonlinear problem, as regards both the gravity current scaling properties and spatial profile of the moving interface.

\section{ACKNOWLEDGMENTS}

D.M.A. is partially supported by National Aeronautics and Space Administration Microgravity Science and Applications Program, the 3M Non-Tenured Faculty Award Program, and the Faculty Summer Research Support Program and the Junior Faculty Award Program in Social Sciences and Sciences of the College of Arts and Sciences at George Mason University. R.M.M. is partially supported by an National Science Foundation (NSF) Career Award, Grant No. DMS-97019242. The efforts of R.M.M. and C.T.M. have been supported in part by Grant No. P42 ES05948 from the National Institute of Environmental Health Sciences and NSF Grant No. DMS-0112069. The computational efforts of C.T.M. were supported by the North Carolina Supercomputer Center.

\section{APPENDIX A: ALTERNATE INTERFACE PARAMETRIZATION}

In the case of a horizontally layered medium with $K_{c}$ $=K_{c}(Z)$ and $Z=z / \epsilon$, we examine a parametrization of the interface position in which it is identified by its horizontal location $x=L(z, Z, t)$. This quantity may have explicit dependence on the fast variable $Z$. In terms of this parametrization the free-surface boundary condition is

$$
\frac{\partial L}{\partial t}+w \frac{\partial L}{\partial z}=u, \quad \text { at } x=L(z, Z, t) .
$$

While this parametrization may be problematic at locations such as the point of symmetry $(x=0)$, where $\partial L / \partial z$ is infinite, it remains an instructive description for the present purposes. If we subject this equation to an expansion in $\epsilon$ coupled to the equations in the bulk as described in Sec. IV C, we find that at leading order in $\epsilon, L_{0}=L_{0}(z, t)$ is independent of the fast variable $Z$ and, furthermore, that $L_{0}$ satisfies

$$
\frac{\partial L_{0}}{\partial t}+\left\langle w_{0}\right\rangle \frac{\partial L_{0}}{\partial z}=\left\langle u_{0}\right\rangle, \quad \text { at } x=L_{0}(z, t),
$$

where in this context $\langle\cdot\rangle$ indicates $\int_{0}^{1} d Z$. The quantities $\left\langle u_{0}\right\rangle$ and $\left\langle w_{0}\right\rangle$ are given by Eqs. (114) and (115).

Under suitable conditions, Eq. (A2) can be expressed back in terms of a function interpreted as height rather than horizontal position. In particular, we assume that $x$ $=L_{0}(z, t)$ defines a function that is invertible so that $z$ $=L_{0}^{-1}(x, t)$ is its inverse function with respect to spatial variables. Here $L_{0}^{-1}(x, t)$ is a height function that we later rename $h_{0}(x, t)$.

The relation $L_{0}^{-1}\left[L_{0}(z, t), t\right]=z$ leads to the following two expressions when taking partial derivatives with respect to $z$ and $t$ :

$$
\begin{aligned}
& \left.\left.\frac{\partial L_{0}^{-1}}{\partial x}\right|_{t} \frac{\partial L_{0}}{\partial z}\right|_{t}=1, \\
& \left.\left.\frac{\partial L_{0}^{-1}}{\partial x}\right|_{t} \frac{\partial L_{0}}{\partial t}\right|_{z}+\left.\frac{\partial L_{0}^{-1}}{\partial t}\right|_{x}=0,
\end{aligned}
$$

where we indicate the quantities that are held fixed during differentiation. If we solve these two equations for $\partial L_{0} / \partial z$ and $\partial L_{0} / \partial t$ and substitute the results into Eq. (A2), we find that 


$$
-\frac{\frac{\partial L_{0}^{-1}}{\partial t}}{\frac{\partial L_{0}^{-1}}{\partial x}}+\left\langle w_{0}\right\rangle\left(L_{0}, z, t\right) \frac{1}{\frac{\partial L_{0}^{-1}}{\partial x}}=\left\langle u_{0}\right\rangle\left(L_{0}, z, t\right),
$$

where we have indicated explicitly the arguments of the two averaged velocities. When $\partial L_{0}^{-1} / \partial x$ is nonzero (which we assume to be true away from the point of symmetry at $x$ $=0$ ), we can rearrange the terms in this equation, use $z$ $=L_{0}^{-1}(x, t)=h_{0}(x, t)$ and $x=L_{0}(z, t)$ to obtain

$$
\frac{\partial h_{0}}{\partial t}+\left\langle u_{0}\right\rangle\left(x, h_{0}, t\right) \frac{\partial h_{0}}{\partial x}=\left\langle w_{0}\right\rangle\left(x, h_{0}, t\right),
$$

which is identical to Eq. (113).

\section{APPENDIX B: IMPLICIT NUMERICAL METHOD}

The governing equations are made dimensionless by scaling lengths with the initial radius $R_{0}$, hydraulic conductivity with $K_{c}^{0}$, and time with $R_{0} / K_{c}^{0}$, where $K_{c}^{0}$ is a reference value for the hydraulic conductivity. The dimensionless equations to solve are

$$
\frac{\partial h}{\partial t}=\frac{\partial}{\partial x}\left[K_{c}(x / \epsilon) h \frac{\partial h}{\partial x}\right],
$$

where $K_{c}=1+K_{0} \cos (2 \pi x / \epsilon)$. Here $K_{0}$ and $\epsilon$ are parameters associated with the permeability function. The evolution equation is subject to the boundary conditions (6) and (7) and the initial conditions

$$
h(x, 0)=A_{H}\left(1-x^{2}\right), \quad R(0)=1,
$$

where $A_{H}$ is a measure of the initial height of the fluid mound that can be related to the initial volume. The contact point $R(t)$ evolves according to

$$
\frac{d R}{d t}=u(R, t)=-\left.K_{c}(R / \epsilon) \frac{\partial h}{\partial x}\right|_{x=R}
$$

We map the horizontal domain $0 \leqslant x \leqslant R(t)$ to the domain $0 \leqslant x^{\prime} \leqslant 1$ using the transformation $x^{\prime}=a_{1}(t) x$ and $t^{\prime}$ $=t$, where

$$
a_{1}(t)=\frac{1}{R(t)} .
$$

It follows that the derivatives transform as

$$
\begin{aligned}
& \frac{\partial}{\partial x} \rightarrow a_{1}(t) \frac{\partial}{\partial x^{\prime}}, \\
& \frac{\partial}{\partial t} \rightarrow \frac{\partial}{\partial t^{\prime}}+\left(\frac{x^{\prime}}{a_{1}\left(t^{\prime}\right)} \frac{d a_{1}}{d t}\right) \frac{\partial}{\partial x^{\prime}},
\end{aligned}
$$

where $d a_{1} / d t=-a_{1}^{2} d R / d t$. This leads to the transformed evolution equation

$$
\frac{\partial h}{\partial t^{\prime}}+\left(\frac{d a_{1}}{d t} \frac{x^{\prime}}{a_{1}\left(t^{\prime}\right)}\right) \frac{\partial h}{\partial x^{\prime}}=a_{1}^{2} \frac{\partial}{\partial x^{\prime}}\left[K_{c} h \frac{\partial h}{\partial x^{\prime}}\right],
$$

subject to

$$
\frac{\partial h}{\partial x}\left(0, t^{\prime}\right)=h\left(1, t^{\prime}\right)=0,
$$

and the initial condition

$$
h(x, 0)=A_{H}\left(1-x^{2}\right) .
$$

Evaluating Eq. (B7) at $x^{\prime}=1$ and enforcing $h=0$ there gives the contact line condition

$$
\frac{d R}{d t}=-\left.\left(K_{c} a_{1} \frac{\partial h}{\partial x}\right)\right|_{x^{\prime}=1} .
$$

This result indicates that Eq. (B7) and $h(R, t)=0$ together determine the motion of the contact line.

The above mapping is convenient because it fixes the computational domain in the horizontal to $0 \leqslant x^{\prime} \leqslant 1$ for all time during the computation. However, one must also keep in mind that since the background permeability variation is fixed in physical space and since the gravity current is spreading the horizontal length scale of the permeability variation in the computational domain $0 \leqslant x^{\prime} \leqslant 1$ is actually getting smaller in time. We need to calculate $K_{c}=1$ $+K_{0} \cos \left(2 \pi R x^{\prime} / \epsilon\right)$, where $x=x^{\prime} R(t)$ at each time step. Therefore, with a fixed horizontal computational grid in $x^{\prime}$, one should have in mind the maximum value of $R$ in order to gauge the resolution needed for a given permeability variation.

We discretize the evolution equation spatially using second-order accurate central differencing with $n x$ grid points in the horizontal direction. The spatial derivative appearing in the evolution equation for $R(t)$ is computed using a one-sided second-order accurate first derivative formula. The time stepping is done implicitly using a backward Euler method. Here, the evolution equations for $h$ and $R$, which take the form

$$
\frac{\partial h}{\partial t}=f\left(x^{\prime}, h, h_{x^{\prime}}, h_{x^{\prime} x^{\prime}}, R, R_{t}\right),
$$

$$
\frac{d R}{d t}=f_{R}\left(x^{\prime}=1, R, h_{x^{\prime}}\right),
$$

are discretized in time as

$$
\begin{aligned}
& -3 h_{j=1}^{i+1}+4 h_{j=2}^{i+1}-h_{j=3}^{i+1}=0, \\
& h_{j}^{i+1}-h_{j}^{i}-\Delta t f^{i+1}=0, \quad \text { for } j=2, \ldots, n x-1, \\
& h_{j=n x}^{i+1}=0, \\
& R^{i+1}-R^{i}-\Delta t f_{R}^{i+1}=0,
\end{aligned}
$$

where 


$$
\begin{aligned}
f^{i+1}= & \left.a_{1}^{i+1} \frac{d K}{d x}\right|_{j} ^{i+1} h_{j}^{i+1} \frac{h_{j+1}^{i+1}-h_{j-1}^{i+1}}{2 \Delta x} \\
& +\left(a_{1}^{i+1}\right)^{2} K_{j}^{i+1} \frac{\left(h_{j+1}^{i+1}-h_{j-1}^{i+1}\right)^{2}}{4 \Delta x^{2}} \\
& +\left(a_{1}^{i+1}\right)^{2} K_{j}^{i+1} h_{j}^{i+1} \frac{h_{j+1}^{i+1}-2 h_{j}^{i+1}+h_{j-1}^{i+1}}{\Delta x^{2}} \\
& +x_{j}^{\prime} f_{R}^{i+1} a_{1}^{i+1} \frac{h_{j+1}^{i+1}-h_{j-1}^{i+1}}{2 \Delta x}, \\
f_{R}^{i+1}= & -K_{j=n x}^{i+1} a_{1}^{i+1} \frac{3 h_{j=n x}^{i+1}-4 h_{j=n x-1}^{i+1}+h_{n x-2}^{i+1}}{2 \Delta x},
\end{aligned}
$$

where $x_{j}^{\prime}=(j-1) /(n x-1), \quad \Delta x=1 /(n x-1), \quad \Delta t=T /(n t$ $-1)$, and $n t$ is the number of temporal points. Note that

$$
\begin{aligned}
& K_{j}^{i+1}=1+K_{0} \cos \left(\frac{2 \pi R^{i+1} x_{j}^{\prime}}{\epsilon}\right), \\
& \left.\frac{d K}{d x}\right|_{j} ^{i+1}=-\frac{2 \pi K_{0}}{\epsilon} \sin \left(\frac{2 \pi R^{i+1} x_{j}^{\prime}}{\epsilon}\right) .
\end{aligned}
$$

Equations (B13)-(B16) represent the symmetry condition, the evolution equation for $h$, the boundary condition $h(R, t)$ $=0$, and the contact line condition (B10). These $n x+1$ expressions are the residuals that are input into a nonlinear solver hybrd.f (which is based on a modification of the Powell hybrid method, and is available in the MINPACK package at NETLIB) to find the unknowns $h_{j}^{i+1}(j=1, \ldots, n x)$ and $R^{i+1}$.

${ }^{1}$ C. T. Miller, G. Christakos, P. T. Imhoff, J. F. McBride, J. A. Pedit, and J. A. Trangenstein, "Multiphase flow and transport modeling in heterogeneous porous media: Challenges and approaches," Adv. Water Resour. 21, 77 (1998).

${ }^{2}$ C. T. Miller, E. H. Hill III, and M. Moutier, "Remediation of DNAPLcontaminated subsurface systems using density-motivated mobilization," Environ. Sci. Technol. 34, 719 (2000).

${ }^{3}$ E. H. Hill III, M. Moutier, J. Alfaro, and C. T. Miller, "Remediation of DNAPL pools using dense-brine barrier strategies," Environ. Sci. Technol. 35, 3031 (2001).
${ }^{4}$ J. Bear, Dynamics of Fluids in Porous Media (Dover, New York, 1972)

${ }^{5}$ G. I. Barenblatt, Scaling, Self-Similarity, and Intermediate Asymptotics (Cambridge University Press, Cambridge, 1996).

${ }^{6}$ I. N. Kochina, N. N. Mikhailov, and M. V. Filinov, "Groundwater mound damping,' Int. J. Eng. Sci. 21, 413 (1983).

${ }^{7}$ T. P. Witelski and A. J. Bernoff, "Self-similar asymptotics for linear and nonlinear diffusion equations," Stud. Appl. Math. 100, 153 (1998).

${ }^{8}$ A. Friedman, B. Hu, and Y. Liu, "A boundary value problem for the Poisson equation with multi-scale oscillating boundary," J. Diff. Eqns. 137, 54 (1997).

${ }^{9}$ A. Friedman and B. Hu, "A non-stationary multi-scale oscillating free boundary for the Laplace and heat equations," J. Diff. Eqns. 137, 119 (1997).

${ }^{10}$ A. Friedman, "Free boundary problems in science and technology," Not. Am. Math. Soc. 47, 854 (2000).

${ }^{11}$ H. E. Huppert, "The propagation of two-dimensional and axisymmetric viscous gravity currents over a rigid horizontal surface," J. Fluid Mech. 121, 43 (1982).

${ }^{12}$ H. E. Huppert and A. W. Woods, "Gravity-driven flows in porous layers," J. Fluid Mech. 292, 55 (1995).

${ }^{13}$ J. M. Acton, H. E. Huppert, and M. G. Worster, "Two-dimensional viscous gravity currents flowing over a deep porous medium,' J. Fluid Mech. 440, 359 (2001).

${ }^{14}$ A. Bensoussan, J. L. Lions, and G. Papanicolaou, "Asymptotic analysis for periodic structures," Studies in Mathematics and its Applications (North-Holland, Amsterdam, 1978), Vol. 5.

${ }^{15}$ J. B. Keller, "Darcy's law for flow in porous media and the two-space method," Nonlinear Partial Differential Equations, in Engineering and Applied Science, edited by R. L. Steinberg, A. J. Kalinowski, and J. S. Papdakis (Marcel Dekker, New York, 1980), pp. 429-443.

${ }^{16}$ D. McLaughlin, G. Papanicolaou, and O. Pironneau, "Convection of microstructure and related problems," SIAM (Soc. Ind. Appl. Math.) J. Appl. Math. 45, 780 (1985).

${ }^{17}$ D. L. Koch and J. F. Brady, "Anomalous diffusion due to long-range velocity fluctuations in the absence of a mean flow," Phys. Fluids A 1, 47 (1989).

${ }^{18}$ A. J. Majda and P. R. Kramer, "Simplified models for turbulent diffusion: Theory, numerical modelling, and physical phenomena," Phys. Rep. 314, 237 (1990).

${ }^{19}$ A. J. Majda and R. M. McLaughlin, "The effect of mean flows on enhanced diffusivity in transport by incompressible periodic velocity fields," Stud. Appl. Math. 89, 245 (1993).

${ }^{20}$ U. Hornung, Homogenization and Porous Media (Springer-Verlag, New York, 1997).

${ }^{21}$ J. Bonn and R. M. McLaughlin, "Sensitive enhanced diffusivities for flows with fluctuating mean winds: A two-parameter study," J. Fluid Mech. 445, 345 (2001).

${ }^{22}$ M. H. Holmes, Introduction to Perturbation Methods (Springer-Verlag, New York, 1995), pp. 236-237. 